\title{
Disturbance-adaptive stochastic optimal control of energy harvesters, with application to ocean wave energy conversion ${ }^{\text {th }}$
}

\author{
J. T. Scruggs ${ }^{\mathrm{a}, *}, \mathrm{R} \cdot \mathrm{Nie}^{\mathrm{a}}$ \\ ${ }^{a}$ University of Michigan, Ann Arbor, MI, USA 48109-2125
}

\begin{abstract}
This paper proposes a technique for optimizing the power generated from stationary stochastic vibratory disturbances, using a resonant energy harvester. Although the theory is general, the target application of the paper concerns ocean wave energy harvesting. The control technique involves the use of a causal discrete-time feedback algorithm to dynamically optimize the power extracted from the waves. The theory assumes that the input impedance of the converter is known precisely, but that a priori models are unavailable for the characterization of the stochastic behavior of the incident waves as well as the transfer functions characterizing their hydrodynamic excitation of the system. For these assumptions, we develop an adaptive control technique, which adapts the feedback law at each time step based on updated estimates for the stochastic disturbance model, obtained through a subspace-based system identification algorithm. The technique is demonstrated on a simulation example pertaining to a cylindrical surface-floating wave energy converter in heave.
\end{abstract}

Keywords: Energy harvesting, ocean wave energy, adaptive regulation, subspace identification

\footnotetext{
A preliminary version of this paper was presented at the 19th IFAC World Congress, Cape Town, August 24-29, 2014.

* Corresponding author

Email addresses: jscruggs@umich.edu (J. T. Scruggs), nie@umich.edu (R. Nie)
}

Preprint submitted to Annual Reviews in Control

September 18, 2015

(C) 2015. This manuscript version is made available under the Elsevier user license http://www.elsevier.com/open-access/userlicense/1.0/ 


\section{Introduction}

It has long been recognized that control theory can be used to optimize the power generated by ocean wave energy converters (Evans, 1981; Falnes, 2002; Salter et al., 2002; Falcaoo, 2010; Ringwood et al., 2014). The determination of the optimal controller for a wave energy converter (WEC) system is predicated on knowledge of its dynamic behavior, as well as a characterization of the sea state to which it is to be subjected. For WECs with linear dynamic models, control designs typically presume harmonic waves, and are designed according to the same network-theoretic impedance-matching principles used in the design and operation of antenna arrays and waveguides (Falnes, 1980). This design technique results in a feedback law between the voltage (or, equivalently, velocity) and the colocated current (or, equivalently, force) of the WEC system, which optimizes power absorption.

However, true sea states are stochastic, with standardized power spectra (such as Pierson-Moskowitz or JONSWAP spectra (Faltinsen, 1990)) which exhibit significant available energy over a nontrivial band of frequencies. For such cases, controllers optimized via impedance matching theory must impose a feedback law which is the Hermitian adjoint (i.e., complex-conjugate transpose) of the driving-point admittance matrix for the WEC, at all frequencies (Nebel, 1992). Such controllers are always anticausal, and thus require some anticipatory technique in which present decisions are made with future wave information.

This can be accomplished with model predictive control (MPC) techniques (Bacelli et al., 2009; Cretel et al., 2011; Li \& Belmont, 2014). Such techniques make use of deployable, up-wave free surface elevation sensors to construct a forecast for future wave excitation forces on the WEC. With this forecast, the optimal control trajectory of the WEC system can then be determined in an anticipatory manner, and can be periodically updated as forecasts are improved. If nonlinear dynamics and constraints require to be taken into account, the MPC techniques can facilitate this via the use of standard Hamilton-Jacobi 
optimal control techniques (Hals et al., 2011; Richter et al., 2013). Although MPC techniques are quite powerful, they generally presume complete and precise knowledge of the system model (including the mapping from the free surface elevation to the resultant excitation forces on the WEC), and that the excitation forces can be predicted accurately over some receding horizon (Fusco \& Ringwood, 2010). The methodology also assumes that the system state (including the dynamic states of the surrounding fluid) can be observed in real time with sufficient accuracy.

In applications where it is desirable to limit the real-time measurements to phenomena in close proximity to the WEC, controllers can alternatively be optimized subject to the constraint of causality. Over the years, a great many causal controllers have been proposed. (See, for example, Hals et al. (2011) and Fusco \& Ringwood (2011), and the references therein). It was recently shown by Scruggs et al. (2013) that under the assumptions of linear dynamics, a stationary stochastic sea state, and unconstrained power controllability, the optimal causal WEC control problem is a special case of the classical Linear Quadratic Gaussian (LQG) control problem. The optimal causal controller has a number of features (besides, of course, causality) that differentiate it from the optimal anticausal controller. Most importantly, while the optimal anticausal controller does not depend on the power spectrum of the sea state, the optimal causal controller does. The reason for this is that the optimal causal controller, operating without the benefit of precise knowledge of future excitations to the system, contains an internal model for the excitation force dynamics. In effect, this internal model can be viewed as providing an optimally-estimated forecast of future excitation forces, based on past data.

In most realistic applications of control to wave energy conversion, it is straight-forward to identify a model for the controllable response of the system (i.e., the mapping from the control force, or current, to the feedback measurements), because both the inputs and output measurements are readily available, and because the control input may be used to probe the system. However, there will be uncertainty about the nature of the wave excitation; both in terms of 
the power spectrum and propagatory direction of the free surface elevation, as well as the mapping between the free surface elevation and the resultant excitation forces. Causal controllers that are optimized under an assumed disturbance model, which is markedly different from the true disturbance, may perform quite poorly - so much so that they may exhibit negative average power generation. It is therefore essential that causal controllers be capable of accommodating disturbance model uncertainties, either through robust control techniques, adaptation, or some combination of both.

In this paper, we consider the design of controllers that are disturbanceadaptive; i.e., which presume a precise a priori model for the controllable response of the system, but for which no a priori knowledge is assumed for the hydrodynamic excitation other than that it is a stochastic process. This approach falls into a class of adaptive control techniques sometimes called adaptive regulation, to imply the situation in which the plant model is assumed to be known, but the controller must be made to adapt to unknown or variable disturbance characteristics (Landau et al., 2011). The approach taken in this paper accomplishes adaptation indirectly; i.e., by identifying a stochastic model for the disturbance, and then re-optimizing the feedback law under the assumption of certainty-equivalence.

\subsection{Scope of the paper}

For the majority of the paper, we develop the theory for general vibration energy harvesting systems (i.e., not for wave energy applications, specifically), as shown in Figure 1. In this diagram, the "passive harvester" is a generic mechanical assemblage (such as a WEC) which is driven to vibrate by an external vibratory disturbance (such as a wave). We assume that within the passive harvester are embedded $n_{p}$ generic "transducers," the ports of which allow energy to flow to and from the harvester. (In the literature on wave energy, these transducers are generally called power take-off systems.) Vectors $i$ and $v \in \mathbb{R}^{n_{p}}$ are the colocated current and voltage vectors for the transducers, $a \in \mathbb{R}^{n_{a}}$ is a vector of disturbances, and $y \in \mathbb{R}^{n_{y}}$ is the vector of feedback outputs. Components 


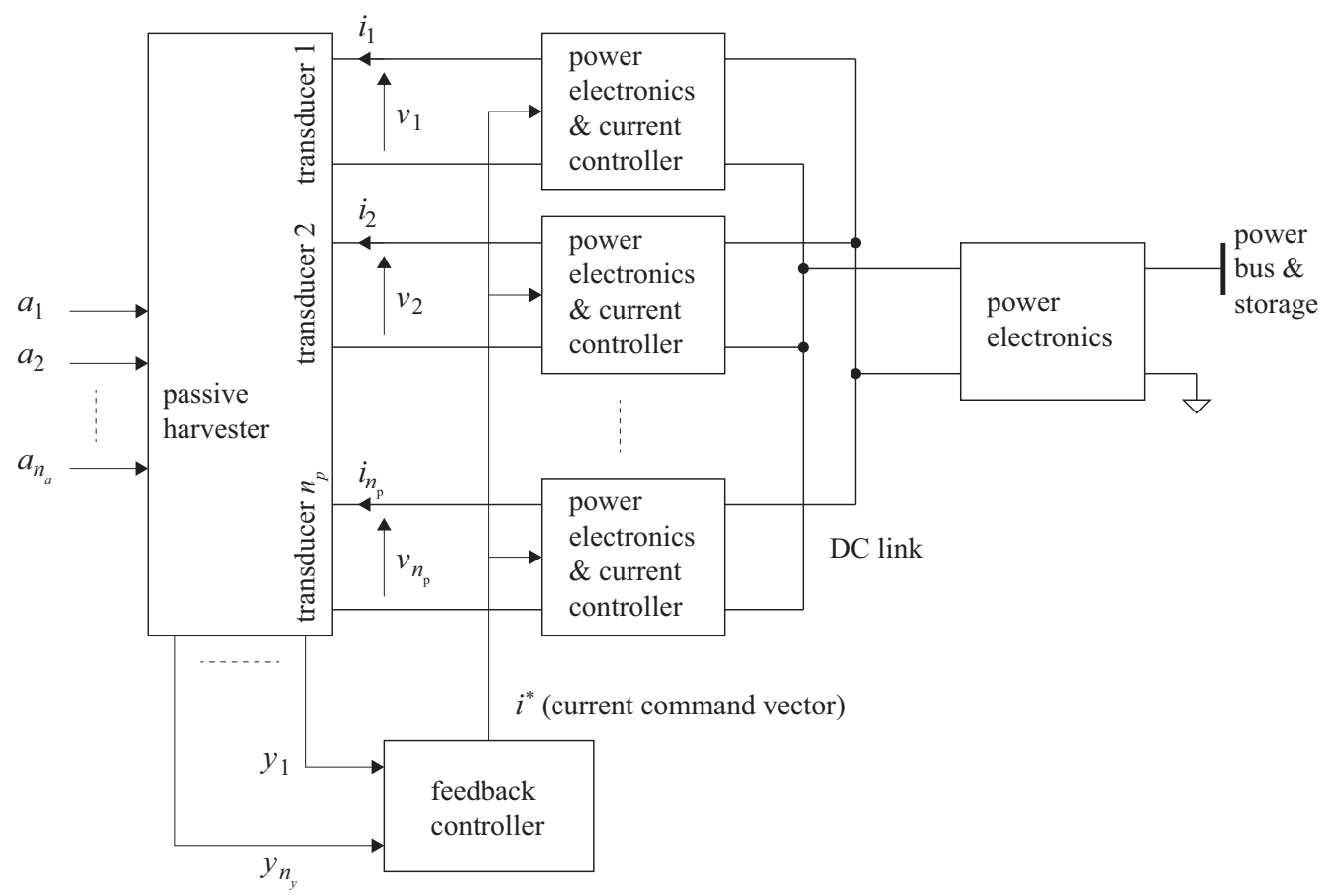

Figure 1: Diagram of a general energy harvesting system

of $y$ may include information about the response states of the harvester, as well as information about disturbance $a$. Components of $a$ may include exogenous disturbances to the system, as well as measurement noise. The feedback controller maps $y$ into a vector $i^{*}$ of desired current commands for each transducer. Each component of $i^{*}$ is then sent to a localized power electronic controller, which is assumed to facilitate high-bandwith current tracking, resulting in the assumption that from the point of view of the harvester dynamics, $i^{*}(t) \approx i(t)$.

With the high-bandwidth current tracking assumption, the system in Figure 1 is approximately equivalent to the block diagram in Figure 2. In this context, the optimal energy harvesting control design problem is to determine the feedback law that maximizes the generated power from the harvester; i.e., the time-averaged value of $-i^{T} v$. For some technologies, such as hydraulic power take-off systems for WECs, it makes more sense to think about control of mechanical colocated quantities instead of electrical quantities. In such cir- 


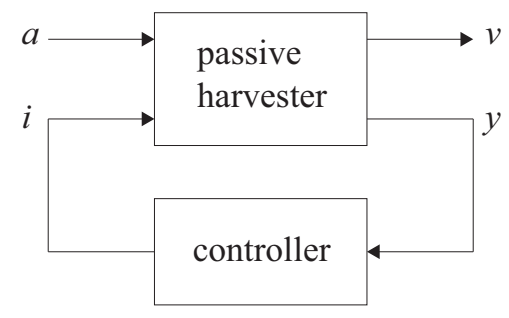

Figure 2: Block diagram of energy harvesting system

cumstances the theory here may still be applied, by taking $i$ to be the force (or torque) vector of the power take-off devices, and $v$ as the colocated linear (or rotational) velocities. There are also other instances in which it may be more reasonable to control voltage (or velocity) rather than current (or force). In these situations the physical meanings of $i$ and $v$ may uniformly be switched throughout the analysis. However, for uniformity of presentation, we will retain our convention as above.

The above formulation implicitly assumes that the power-electronic system is highly efficient, such that the power absorbed from the transducers is approximately equivalent to the power delivered to the bus and storage systems in Figure 1. However, the control objective, as we have stated it, can be modified to accommodate electronic transmission losses by redefining $v$ to include effective voltage drops associated with this dissipation. This has been illustrated by Scruggs et al. (2012), and casts the energy harvesting problem in a more generalized and realistic context. However, the present paper does not explicitly consider electronic transmission losses in the control designs, as it adds an extra layer of complexity and distracts from the paper's primary objective. It is nonetheless straight-forward to extend the disturbance-adaptive control methods discussed here to accommodate various transmission loss models.

We exclusively consider discrete-time implementations, in which $i(t)$ is implemented via a zero-order-hold mapping from its discrete time samples. We will also uniformly assume $a(t)$ to be a stationary stochastic process. However, causal energy harvesting problems in other contexts are also straight-forward to 
analyze. For example, the continuous-time case follows analogously (Scruggs, 2010; Scruggs et al., 2012), and the problem where $a(t)$ is modeled as an impulsive disturbance is discussed in (Scruggs \& Behrens, 2011). Here, we choose a discrete-time, stationary stochastic context because it most readily lends itself to the disturbance-adaptive techniques we present in Section 4.

The primary objective of the paper is to provide a theoretical foundation for disturbance-adaptive optimal causal energy harvesting control, in the context described above. To do this, an effort has been made to be as explicit as possible about the modeling assumptions (such as linearity) that must be made in order for the theory to hold rigorously. However, in practice many of these assumptions will clearly only hold, at best, in some approximate sense. For example, in WEC applications $a(t)$ is not strictly stationary, as its spectrum is constantly changing. Similarly, WEC dynamics always exhibit nonlinearities which must be taken into account in order for simulations to be accurate and control designs to be truly "optimal." Moreover, WEC systems have constraints that limit the feasibility domain for displacements and control forces. In such cases, the methods described here might be extended, but with the caveat that the proofs will not hold rigorously. For example, regarding the assumption of linear dynamics, the design technique proposed here can be applied using the linearized model arising from the small-wave assumption, or through the use of statistical linearization (Scruggs et al., 2015).

\subsection{Notation}

The notation used throughout the paper is mostly standard. However, here we note some terms that may not be entirely commonplace. The sets $\mathbb{R}_{\geq 0}$ and $\mathbb{Z}_{\geq 0}$ refer to the sets of nonnegative real numbers and integers, respectively. We refer to a matrix $A \in \mathbb{R}^{n \times n}$ as Schur, if each of its eigenvalues has modulus less than 1. The notation $\operatorname{tr}\{A\}$ refers to the trace of $A$. For a Hermitian matrix $Q$, we use notation $Q>0$ and $Q \geqslant 0$ to denote positive definiteness and positive semidefiniteness, respectively. Analogous notation is used for negative definiteness and semidefiniteness. For a matrix $A$, we denote the transpose and 
complex-conjugate-transpose as $A^{T}$ and $A^{H}$, respectively. For a vector $x$ and (Hermitian) matrix $Q>0$ of compatible dimension, $\|x\|_{Q}^{2}=x^{H} Q x$. For a random variable $u$, we denote its expectation as $\mathcal{E}\{u\}$. If $u(t)$ is an ergodic stochastic process on $t \in \mathbb{R}$ or $\mathbb{Z}$, then $\mathcal{E}\{u\}$ implies that the expectation is taken in stationarity, with the time and ensemble averages being equal.

\section{Problem formulation}

\subsection{Preliminaries}

We assume the forward-loop continuous-time dynamics illustrated in Figure 2 can be represented by a linear model, represented in the Laplace domain as

$$
\left[\begin{array}{l}
\hat{v}(s) \\
\hat{y}(s)
\end{array}\right]=\left[\begin{array}{ll}
Z_{i v}(s) & Z_{a v}(s) \\
Z_{i y}(s) & Z_{a y}(s)
\end{array}\right]\left[\begin{array}{l}
\hat{i}(s) \\
\hat{a}(s)
\end{array}\right]
$$

where we assume the above transfer function matrix is in $\mathcal{H}_{\infty}$. We assume $a(t)$ to be a stationary stochastic process characterized by an autocorrelation function $R_{a}(\tau)=\mathcal{E}\left\{a(t+\tau) a^{T}(t)\right\}$ and a corresponding power spectral density

matrix $S_{a}(\omega)=\int_{-\infty}^{\infty} R_{a}(\tau) e^{-j \omega \tau} d \tau$. We assume that $a(t)$ has finite variance in stationary response, and consequently, that $\int_{-\infty}^{\infty}\left\|S_{a}(\omega)\right\|^{2} d \omega<\infty$.

Considering the transfer function $Z_{i v}(s)$, we see that this is the driving point impedance of the harvester, as seen from the transducer port terminals. In order for the energy harvesting problem to be well-posed, it must be the case that the harvesting system has no internal energy sources. (Otherwise the optimal control of $i(t)$ will be destabilizing.) Consequently, we assume that the mapping $i \mapsto v$ is passive. Together with our assumption of linearity, this implies that $Z_{i v}(s)$ is positive-real (Anderson \& Vongpanitlerd, 1973); i.e., that it is analytic for $\Re\{s\}>0$, real for real $s>0$, and

$$
Z_{i v}(s)+Z_{i v}^{H}(s) \geqslant 0 \quad, \quad \forall \Re\{s\} \geqslant 0
$$

In this paper we consider exclusively a discrete-time treatment of the energy harvesting problem. We presume that the current $i(t)$ is controlled via a zero- 
order-hold D/A conversion; i.e.,

$$
i(t)=i_{k} \quad, \quad t \in \mathcal{T}_{k}
$$

where $\mathcal{T}_{k}=[k T,(k+1) T)$ and where $T$ is the sample time. We will use the same notational convention for other sampled signals; i.e., $y_{k}=y(k T)$, etc.

Let $q(t)$ be the moving average of $v(t)$ over retrospective time interval $T$; i.e.,

$$
q(t)=\frac{1}{T} \int_{t-T}^{t} v(\tau) d \tau
$$

and let $q_{k}$ be its associated sample sequence. Then the average power generated during time period $t \in \mathcal{T}_{k}$ is

$$
p_{k}=-i_{k}^{T} q_{k+1}
$$

We presume that the dynamic behavior of the energy harvesting system is inferred directly from observed discrete-time response data, and without direct knowledge of the continuous-time model. As such, attainment of the maximum harvested energy requires that $q_{k}$ be measurable directly, at least for the purpose of discrete-time system identification. In this paper, we assume that $q_{k}$ can be measured directly, although possibly corrupted by independent, identicallydistributed (iid) measurement noise, and that its noise-corrupted version is contained in $y_{k}$; i.e., that there exists a known matrix $E_{y q}$ such that

$$
q_{k}=E_{y q} y_{k}+\eta_{k}
$$

where $\eta_{k}$ is the zero-mean noise sequence with covariance

$$
\begin{aligned}
& \mathcal{E} \eta_{k} y_{\ell}^{T}=0 \\
& \mathcal{E} \eta_{k} \eta_{\ell}^{T}=\Sigma_{\eta} \delta_{k \ell}
\end{aligned}
$$

for $\Sigma_{\eta} \geqslant 0$, and where $\delta_{k \ell}$ is the Kronecker delta.

The continuous-time system with the assumptions above can be converted into an equivalent system in discrete time, as

$$
\hat{y}(z)=G_{i y}(z) \hat{i}(z)+\hat{y}_{0}(z)
$$


where $G_{i y}\left(e^{j \Omega}\right)$ can be expressed as

$$
G_{i y}\left(e^{j \Omega}\right)=\left.\lim _{\epsilon \rightarrow 0} \sum_{\ell=-\infty}^{\infty} Z_{i y}(j \omega) \operatorname{sn}(\omega T / 2) e^{j(-\omega T / 2+\epsilon)}\right|_{\omega=(\Omega+2 \pi \ell) / T}
$$

where $\operatorname{sn}(u)=\sin (u) / u$ is the sinc function, and $\Omega \in(-\pi, \pi]$ is the discrete-time frequency. In the above, we have used the fact that, for all $\epsilon \in(0, T)$,

$$
1=\left.\sum_{\ell=-\infty}^{\infty} e^{j \omega \epsilon} \operatorname{sn}(\omega T / 2) e^{-j \omega T / 2}\right|_{\omega=(\Omega+2 \pi \ell) / T}
$$

to accommodate cases in which $Z_{i y}(s)$ is not strictly proper. Transfer function $G_{i y}(z)$ is then the analytic continuations of $G_{i y}\left(e^{j \Omega}\right)$ for $z=e^{j \Omega}$.

Let $G_{i q}(z)$ be the discrete-time transfer function from $i_{k}$ to $q_{k}$, equal to

$$
G_{i q}(z)=E_{y q} G_{i y}(z) .
$$

It is straight-forward to show that $G_{i q}\left(e^{j \Omega}\right)$ may equivalently be related directly to $Z_{i v}(s)$ via

$$
\left.G_{i q}\left(e^{j \Omega}\right)=e^{-j \Omega}\left[\sum_{\ell=-\infty}^{\infty} Z_{i v}(j(\Omega+2 \pi \ell) / T)\right) \operatorname{sn}^{2}(\Omega / 2+\pi \ell)\right]
$$

In order for the optimal energy harvesting control problem to be well-posed it will be necessary that the transfer function from $i_{k}$ to $q_{k+1}$ (i.e., $\left.z G_{i q}(z)\right)$ be positive-real; i.e., that

$$
e^{j \Omega} G_{i q}\left(e^{j \Omega}\right)+e^{-j \Omega} G_{i q}^{T}\left(e^{-j \Omega}\right) \geqslant 0 \quad, \quad \forall \Omega \in(-\pi, \pi]
$$

From (13), we see that this condition is guaranteed if $Z_{i v}(s)$ is positive-real; i.e., satisfies (2).

The discrete-time sequence $y_{0 k}$ is the uncontrolled (i.e., "open circuit") response for $y_{k}$, and is thus modeled as a stochastic processes with a spectrum arising from the sampled continuous-time process. In other words, for the continuous-time open-circuit autocorrelation

$$
R_{y_{0}}(\tau)=\left.\mathcal{E}\left\{y(t+\tau) y^{T}(t)\right\}\right|_{i(t)=0, \forall t}
$$


its corresponding continuous-time power spectrum is

$$
S_{y_{0}}(\omega)=Z_{a y}(j \omega) S_{a}(\omega) Z_{a y}^{T}(-j \omega)
$$

The autocorrelation associated with the discrete-time sampled process is then $R_{y_{0}}(k T)$, and the corresponding discrete-time spectrum, which we denote $\Sigma_{y_{0}}(\Omega)$ is then

$$
\begin{aligned}
\Sigma_{y_{0}}(\Omega) & =\sum_{k=-\infty}^{\infty} R_{y_{0}}(k T) e^{-j \Omega k} \\
& =\left.\frac{1}{T} \sum_{\ell=-\infty}^{\infty} S_{y_{0}}(\omega)\right|_{\omega=(\Omega+2 \pi \ell) / T}
\end{aligned}
$$

\subsection{Finite-dimensional system model}

In order to create a tractable problem, we now make the assumption that the discrete-time system in (9), together with $\Sigma_{y_{0}}(\Omega)$ in (18), can be approximated by the finite-dimensional, forward-time, Gauss-Markov innovations model

$$
\begin{aligned}
{\left[\begin{array}{c}
x_{1, k+1} \\
x_{2, k+1}
\end{array}\right] } & =\left[\begin{array}{cc}
A_{11} & A_{12} \\
0 & A_{22}
\end{array}\right]\left[\begin{array}{l}
x_{1, k} \\
x_{2, k}
\end{array}\right]+\left[\begin{array}{c}
B_{i 1} \\
0
\end{array}\right] i_{k}+\left[\begin{array}{c}
B_{e 1} \\
B_{e 2}
\end{array}\right] e_{k} \\
y_{k} & =\left[\begin{array}{ll}
C_{y 1} & C_{y 2}
\end{array}\right]\left[\begin{array}{l}
x_{1, k} \\
x_{2, k}
\end{array}\right]+D_{i y} i_{k}+e_{k}
\end{aligned}
$$

where $x_{k} \in \mathbb{R}^{n}$ is the finite-dimensional state, and $e_{k} \in \mathbb{R}^{n_{y}}$ is the innovations sequence, characterized as a white noise sequence with zero mean and $\mathcal{E} e_{k} e_{\ell}^{T}=$ $\Sigma_{e} \delta_{k \ell}$. The finite-dimensional model above also inherits (6), relating $y_{k}$ to $q_{k}$. Note that, because $i \mapsto q$ is strictly causal, we have that $E_{y q} D_{i y}=0$; i.e.,

$$
q_{k}=E_{y q}\left[\begin{array}{ll}
C_{y 1} & C_{y 2}
\end{array}\right]\left[\begin{array}{c}
x_{1, k} \\
x_{2, k}
\end{array}\right]+E_{y q} e_{k}+\eta_{k}
$$

When we state that the above model "approximates" the true system, we mean that to adequate precision, the power spectral density $\Sigma_{y_{0}}(\Omega)$ as in (18) is related to the state space parameters via

$$
\Sigma_{y_{0}}(\Omega)=U\left(e^{j \Omega}\right) \Sigma_{e} U^{H}\left(e^{j \Omega}\right)
$$


where $U(z)$ is the spectral factor

$$
U(z)=C_{y}[z I-A]^{-1} B_{e}+I
$$

and that $G_{i y}(z)$ is

$$
G_{i y}(z)=C_{y}[z I-A]^{-1} B_{i}+D_{i y}
$$

In order to reduce the level of formalism required to present the results in this paper, the above equalities will henceforth be assumed to hold precisely. However, it is worth noting that the above model will often be at best an approximation of the true system, even if the harvester dynamics are linear and finite-dimensional, because $S_{a}(\omega)$ is often (as in the case of wave energy applications) an irrational spectrum.

In the above model, we make the following assumptions:

(A1) The above is a minimal realization, and $A$ is Schur.

(A2) $x_{1}$ is the largest subspace of $x \in \mathbb{R}^{n}$ that is controllable from $i$.

(A3) $U(z)$, as defined in (22), is minimum-phase.

(A4) The transfer function

$$
z G_{i q}(z)=z E_{y q} C_{y}[I z-A]^{-1} B_{i}
$$

is strictly positive-real; i.e., there exists some $\beta \in(0,1)$ such that it is analytic for all $|z| \geqslant \beta$, and such that

$$
\beta e^{j \Omega} G_{i q}\left(\beta e^{j \Omega}\right)+\beta e^{-j \Omega} G_{i q}^{T}\left(\beta e^{-j \Omega}\right)>0
$$

Regarding these assumptions, we have the following remarks and justifications:

- Assumption (A1) is reasonable, because if $A$ is not asymptotically stable, the optimal energy harvesting controller will not result in a stabilized closed-loop system unless additional constraints are imposed on the optimization. Although harvesting energy from unstable dynamical systems is an interesting area of study, it is outside the scope of this paper. 
- Assumption (A2) is merely by convention, and can always be achieved through an appropriate change of basis. The assumption leads to the special structure of the $A$ and $B$ matrices.

- Assumption (A3) is a defining feature of an innovations model, and a model as above which violates this assumption can always be converted into an innovations model. Note that because we assume $A$ is asymptotically stable (per Assumption (A1)) this implies that when the model is known precisely, $x_{k}$ can be determined precisely in stationarity, given past values of $i_{k}$ and $y_{k}$; i.e., via the observer

$$
x_{k+1}=\left[A-B_{e} C_{y}\right] x_{k}+\left[B_{i}-B_{e} D_{i y}\right] i_{k}+B_{e} y_{k}
$$

where the minimum-phase condition assures that $A-B_{e} C_{y}$ is Schur.

- If Assumption (A4) had only required that $z G_{i q}(z)$ be positive real (but not strictly so), this would merely be an assurance that the property (14), which is known to be true for the original linear system model, has not been compromised by finite-dimensional approximation. Strengthening the condition to strict positive-realness is not overly restrictive, and is usually justifiable on physical grounds. While strict positive-realness is not technically required in order to conduct the ensuing analysis, it regularizes the optimal control problem to be solved, resulting in a considerably simpler development.

The full problem data, as it pertains to the optimal causal energy harvesting control problem, will be denoted by $\mathcal{M}$, and is comprised of the following parameters:

$$
\mathcal{M}=\left\{A_{11}, A_{12}, A_{22}, B_{i 1}, B_{e}, C_{y 1}, C_{y 2}, D_{i y}, \Sigma_{e}, E_{y q}\right\}
$$

\subsection{Causal energy harvesting control objective}

In terms of the finite-dimensional model parameters, we have that the generated power during interval $\mathcal{T}_{k}$ is

$$
p_{k}=-i_{k}^{T}\left(E_{y q} C_{y} x_{k+1}+E_{y q} e_{k+1}+\eta_{k+1}\right)
$$


We wish to maximize the mean value of $p_{k}$ in stationary response, thus arriving at the maximization objective

$$
\bar{p}=-\mathcal{E}\left\{i_{k}^{T} E_{y q} C_{y} x_{k+1}+i_{k}^{T} E_{y q} e_{k+1}+i_{k}^{T} \eta_{k+1}\right\}
$$

Define the sets

$$
\begin{aligned}
& \mathcal{Y}_{k}=\left\{y_{k-1}, y_{k-2}, \ldots\right\} \\
& \mathcal{I}_{k}=\left\{i_{k-1}, i_{k-2}, \ldots\right\}
\end{aligned}
$$

In the discrete-time causal control formulation, the feedback system maps $\left\{\mathcal{Y}_{k}, \mathcal{I}_{k}\right\} \mapsto$ $i_{k}$. And from Assumption A3, $x_{k}$ is known precisely, given $\left\{\mathcal{Y}_{k}, \mathcal{I}_{k}, \mathcal{M}\right\}$. Consequently,

$$
\begin{aligned}
\mathcal{E}\left\{i_{k}^{T} E_{y q} C_{y} x_{k+1} \mid \mathcal{Y}_{k}, \mathcal{I}_{k}, \mathcal{M}\right\} & =i_{k}^{T} E_{y q} C_{y}\left(A x_{k}+B_{i} i_{k}\right) \\
\mathcal{E}\left\{i_{k}^{T}\left(E_{y q} e_{k+1}+\eta_{k+1}\right) \mid \mathcal{Y}_{k}, \mathcal{I}_{k}, \mathcal{M}\right\} & =0
\end{aligned}
$$

Thus we have that

$$
\mathcal{E}\left\{p_{k} \mid \mathcal{Y}_{k}, \mathcal{I}_{k}, \mathcal{M}\right\}=-i_{k}^{T} C_{p} x_{k}-i_{k}^{T} D_{p} i_{k}
$$

where

$$
\begin{aligned}
& C_{p}=E_{y q} C_{y} A \\
& D_{p}=\frac{1}{2}\left(E_{y q} C_{y} B_{i}+B_{i}^{T} C_{y}^{T} E_{y q}^{T}\right)
\end{aligned}
$$

We therefore have that the causal optimization objective is

$$
\begin{aligned}
\bar{p} & =\mathcal{E}\left\{\mathcal{E}\left\{p_{k} \mid \mathcal{Z}_{k}, \mathcal{I}_{k}, \mathcal{M}\right\}\right\} \\
& =-\mathcal{E}\left\{i_{k}^{T} C_{p} x_{k}+i_{k}^{T} D_{p} i_{k}\right\}
\end{aligned}
$$

Regarding this objective, we make the following remarks:

- Note that the causal energy harvesting performance objective does not require that the process $\eta_{k}$ in (6) be characterized, beyond the requirement that it be iid with zero mean and finite variance. As such, it is not necessary to know or assume $\Sigma_{\eta}$, as it does not show up either in the discrete-time model, or the optimization objective. 
- It will be useful to state explicitly the way $C_{p}$ and $D_{p}$ depend on the partitioned parameters of subspaces $x_{1}$ and $x_{2}$. Specifically, we have that

$$
\begin{aligned}
C_{p 1} & =E_{y q} C_{y 1} A_{11} \\
C_{p 2} & =E_{y q} C_{y 1} A_{12}+E_{y q} C_{y 2} A_{22} \\
D_{p} & =\frac{1}{2}\left(E_{y q} C_{y 1} B_{i 1}+B_{i 1}^{T} C_{y 1}^{T} E_{y q}^{T}\right)
\end{aligned}
$$

which illustrates the point that $C_{p 1}$ and $D_{p}$ depend only on the parameters of the controllable subspace.

- It is straight-forward to verify that Assumption (A4) is equivalent to the requirement that

$$
G_{p}(z) \triangleq C_{p 1}\left[z I-A_{11}\right]^{-1} B_{i 1}+D_{p}
$$

is strictly positive real.

\section{Optimal causal discrete-time energy harvesting}

In this section we consider the case where $\mathcal{M}$ is considered to be known precisely, e.g., via system identification. We are then interested in deriving the optimal discrete-time feedback law $\left\{\mathcal{Y}_{k}, \mathcal{I}_{k}, \mathcal{M}\right\} \mapsto i_{k}$ which maximizes $\bar{p}$. Toward this end, we first present the following variant of the Generalized Positive Real Lemma (Xiao \& Hill, 1999).

Lemma 1. For the system in (19), and adhering to Assumptions (A1), (A2), and (A4), there exists a unique matrix $W=W^{T}$, partitioned as

$$
W=\left[\begin{array}{ll}
W_{11} & W_{12} \\
W_{12}^{T} & W_{22}
\end{array}\right]
$$

where $W_{11} \in \mathbb{R}^{n_{1} \times n_{1}}$ and $n_{1}$ is the dimension of the controllable subspace, such that

$$
\begin{aligned}
0 & <W_{11} \\
0 & <D_{p}-B_{i}^{T} W B_{i} \\
W & =A^{T} W A+\left(\frac{1}{2} C_{p}^{T}-A^{T} W B_{i}\right)\left(D_{p}-B_{i}^{T} W B_{i}\right)^{-1}\left(\frac{1}{2} C_{p}-B_{i}^{T} W A\right)
\end{aligned}
$$


and for which the matrix

$$
\check{A}=A-B_{i}\left(D_{p}-B_{i}^{T} W B_{i}\right)^{-1}\left(\frac{1}{2} C_{p}-B_{i}^{T} W A\right)
$$

is Schur. Furthermore, this is the solution to (46) which is is minimal in $W_{11}$.

Proof. From the Positive Real Lemma, we have that Assumption (A4) is true if and only if the Riccati equation

$$
\begin{aligned}
W_{11}=A_{11}^{T} W_{11} A_{11}+\left(\frac{1}{2} C_{p 1}^{T}-A_{11}^{T} W_{11} B_{i 1}\right)\left(D_{p}\right. & \left.-B_{i 1}^{T} W_{11} B_{i 1}\right)^{-1} \\
& \times\left(\frac{1}{2} C_{p 1}-B_{i 1}^{T} W_{11} A_{11}\right)
\end{aligned}
$$

has a minimal solution satisfying (44) and $D_{p}>B_{i 1}^{T} W_{11} B_{i 1}$, and furthremore that this solution is the unique solution to (48) for which

$$
\check{A}_{11}=A_{11}-B_{i 1}\left(D_{p}-B_{i 1}^{T} W_{11} B_{i 1}\right)^{-1}\left(\frac{1}{2} C_{p 1}-B_{i 1}^{T} W_{11} A_{11}\right)
$$

is Schur. Now, separate equation $(46)$ into $(1,1),(1,2)$, and $(2,2)$ components analogously to $W$. Observing Assumption (A2), the (1,1) component is (48), and if $W_{11}$ stabilizes $\check{A}_{11}$, then it follows from Assumptions (A1) and (A2) that the solution to (46) minimizing $W_{11}$ results in $\check{A}$ Schur. Additionally, due to Assumption (A2), $B_{i}^{T} W B_{i}=B_{i 1}^{T} W_{11} B_{i 1}$, thus assuring (45). Component $(1,2)$ of equation (46) is

$$
\begin{aligned}
W_{12}= & A_{11}^{T}\left(W_{11} A_{12}+W_{12} A_{22}\right) \\
+ & \left(\frac{1}{2} C_{p 1}^{T}-A_{11}^{T} W_{11} B_{i 1}\right)\left(D_{p}-B_{i 1}^{T} W_{11} B_{i 1}\right)^{-1} \\
& \times\left(\frac{1}{2} C_{p 2}^{T}-B_{i 1}^{T}\left(W_{11} A_{12}+W_{12} A_{22}\right)\right)
\end{aligned}
$$

which can be solved for $W_{12}$ once $W_{11}$ is known. Grouping together all terms that do not multiply by $W_{12}$ into a matrix $Q_{12}$, this equation is a Sylvester equation of the form $W_{12}=\check{A}_{11}^{T} W_{12} A_{22}+Q_{12}$, which has a unique solution if both $\check{A}_{11}$ and $A_{22}$ are Schur. But the former was shown to be true by the development above, resulting from Assumption (A4), while the latter is known to be true by combining Assumptions (A1) and (A2). Finally, the $(2,2)$ component 
of $(46)$ is

$$
\begin{aligned}
W_{22}= & A_{12}^{T} W_{11} A_{12}+A_{12}^{T} W_{12} A_{22}+A_{22}^{T} W_{12}^{T} A_{12}+A_{22}^{T} W_{22} A_{22} \\
+ & \left(\frac{1}{2} C_{p 2}^{T}-A_{12}^{T} W_{11} B_{i 1}-A_{22}^{T} W_{12}^{T} B_{i 1}\right)\left(D_{p}-B_{i 1}^{T} W_{11} B_{i 1}\right)^{-1} \\
& \times\left(\frac{1}{2} C_{p 2}-B_{i 1}^{T} W_{11} A_{12}-B_{i 1}^{T} W_{12} A_{22}\right)
\end{aligned}
$$

Again grouping together all terms not depending on $W_{22}$ into a matrix $Q_{22}$, this equation is a discrete-time Lyapunov equation of the form $W_{22}=Q_{22}+$ $A_{22}^{T} W_{22} A_{22}$ which, because $A_{22}$ is Schur, is guaranteed to give a unique solution for $W_{22}$.

With this lemma, we can now present the main theorem.

Theorem 1. Let assumptions (A1)-(A4) hold, and let $\phi:\left\{\mathcal{Y}_{k}, \mathcal{I}_{k}, \mathcal{M}\right\} \rightarrow i_{k}$ be any feedback law resulting in a wide-sense stationary closed-loop response. Then

$$
\bar{p}=\bar{p}_{0}-\mathcal{E}\|i-K x\|_{\Delta}^{2}
$$

where

$$
\begin{aligned}
\bar{p}_{0} & =\operatorname{tr}\left\{B_{e}^{T} W B_{e} \Sigma_{e}\right\} \\
\Delta & =D_{p}-B_{i}^{T} W B_{i}>0 \\
K & =-\Delta^{-1}\left[\frac{1}{2} C_{p}-B_{i}^{T} W A\right]
\end{aligned}
$$

where $W=W^{T}$ is the solution to (46) which minimizes $W_{11}$. Furthermore, the optimal state feedback law $\phi(x)=K x$ results in a Schur closed-loop dynamics matrix $A+B_{i} K$.

Proof. In wide-sense stationary response, we have that

$$
\bar{p}=-\mathcal{E}\left\{\left[\begin{array}{l}
x \\
i
\end{array}\right]^{T}\left[\begin{array}{cc}
0 & \frac{1}{2} C_{p}^{T} \\
\frac{1}{2} C_{p} & D_{p}
\end{array}\right]\left[\begin{array}{l}
x \\
i
\end{array}\right]\right\}
$$

Now, for any $P=P^{T}$,

$$
\mathcal{E}\left\{x_{k+1}^{T} P x_{k+1}\right\}=\mathcal{E}\left\{\left(A x_{k}+B_{i} i_{k}+B_{e} e_{k}\right)^{T} P\left(A x_{k}+B_{i} i_{k}+B_{e} e_{k}\right)\right\}
$$


But since $e_{k}$ is zero-mean and uncorrelated with $x_{k}$ and $i_{k}$,

$$
\mathcal{E}\left\{x_{k+1}^{T} P x_{k+1}\right\}=\mathcal{E}\left\{\left(A x_{k}+B_{i} i_{k}\right)^{T} P\left(A x_{k}+B_{i} i_{k}\right)\right\}+\operatorname{tr}\left\{B_{e}^{T} P B_{e} \Sigma_{e}\right\}
$$

In stationarity, $\mathcal{E}\left\{x_{k+1}^{T} P x_{k+1}\right\}=\mathcal{E}\left\{x_{k}^{T} P x_{k}\right\}$ so in this case we have that

$$
0=\mathcal{E}\left\{\left[\begin{array}{l}
x \\
i
\end{array}\right]^{T}\left[\begin{array}{cc}
A^{T} P A-P & A^{T} P B_{i} \\
B_{i}^{T} P A & B_{i}^{T} P B_{i}
\end{array}\right]\left[\begin{array}{l}
x \\
i
\end{array}\right]\right\}+\operatorname{tr}\left\{B_{e}^{T} P B_{e} \Sigma_{e}\right\}
$$

Subtracting this from (56) we get that

$$
\bar{p}=-\mathcal{E}\left\{\left[\begin{array}{l}
x \\
i
\end{array}\right]^{T}\left[\begin{array}{cc}
A^{T} P A-P & \frac{1}{2} C_{p}^{T}+A^{T} P B_{i} \\
\frac{1}{2} C_{p}+B_{i}^{T} P A & D_{p}+B_{i}^{T} P B_{i}
\end{array}\right]\left[\begin{array}{l}
x \\
i
\end{array}\right]\right\}-\operatorname{tr}\left\{B_{e}^{T} P B_{e} \Sigma_{e}\right\}
$$

Via a Schur complement, this is equivalent to

$$
\bar{p}=-\mathcal{E}\left\{\left[\begin{array}{c}
x \\
i-K x
\end{array}\right]^{T}\left[\begin{array}{ll}
\Theta & 0 \\
0 & \Delta
\end{array}\right]\left[\begin{array}{c}
x \\
i-K x
\end{array}\right]\right\}-\operatorname{tr}\left\{B_{e}^{T} P B_{e} \Sigma_{e}\right\}
$$

where

$$
\Theta=A^{T} P A-P-\left(\frac{1}{2} C_{p}^{T}+A^{T} P B_{i}\right)\left(D_{p}+B_{i}^{T} P B_{i}\right)^{-1}\left(\frac{1}{2} C_{p}+B_{i}^{T} P A\right)
$$

and where $K=-\Delta^{-1}\left(\frac{1}{2} C_{p}+B_{i}^{T} P A\right)$ and $\Delta=D_{p}+B_{i}^{T} P B_{i}$. Finally, set $P=-W$, the solution to (46), which makes $\Theta=0, \Delta>0$, and guarantees that $A+B_{i} K$ is Schur. With this substitution, (61) becomes (52).

Due to Assumption (A2), the solutions to $W$ and $K$ have special structure. Let $K$ be partitioned into parameters for the controllable and uncontrollable subspaces, as

$$
K=\left[\begin{array}{ll}
K_{1} & K_{2}
\end{array}\right]
$$

From the proof of Lemma 1 we know that $W_{11}, K_{1}$, and $\Delta$ can be solved independently of $W_{12}$ and $W_{22}$, as

$$
\begin{aligned}
W_{11} & =A_{11}^{T} W_{11} A_{11}+K_{1}^{T} \Delta K_{1} \\
K_{1} & =-\Delta^{-1}\left[\frac{1}{2} C_{p 1}-B_{i 1}^{T} W_{11} A_{11}\right] \\
\Delta & =D_{p}-B_{i 1}^{T} W_{11} B_{i 1}
\end{aligned}
$$


With these terms solved, $W_{12}$ is solved via a (linear) Sylvester equation:

$$
W_{12}=\check{A}_{11}^{T} W_{12} A_{22}+\check{A}_{11}^{T} W_{11} A_{12}-\frac{1}{2} K_{1}^{T} C_{p 2}
$$

where $\check{A}_{11}=A_{11}+B_{i 1} K_{1}$. With $W_{12}$ found, $K_{2}$ is a linear function of it, as

$$
K_{2}=-\Delta^{-1}\left[\frac{1}{2} C_{p 2}-B_{i 1}^{T}\left(W_{11} A_{12}+W_{12} A_{22}\right)\right]
$$

Note that to determine $K_{1}$ and $K_{2}$, it is not necessary to find $W_{22}$ explicitly, unless it is required to solve for $\bar{p}_{0}$.

Theorem 1 is useful because it converts the energy harvesting control problem into a tracking problem. The objective is to minimize the mean value of $\|i-K x\|_{\Delta}^{2}$ in stationarity. In the absence of model uncertainty or competing control design constraints, it also gives us the physical limit on power generation (with causal, discrete-time feedback), and the controller that achieves it.

Corollary 1. For assumptions (A1)-(A4), assume $\mathcal{M}$ is known precisely. Then the maximum power generation achievable by a causal controller $\phi:\left\{\mathcal{Y}_{k}, \mathcal{I}_{k}, \mathcal{M}\right\} \rightarrow$ $i_{k}$ is $\bar{p}_{0}$, as found in (53). The controller that achieves it is unique, linear, and of the form $\hat{i}(z)=\Phi(z) \hat{y}(z)$ where

$$
\Phi(z)=K\left[z I-\left(A+B_{i} K-B_{e} C_{y}-B_{e} D_{i y} K\right)\right]^{-1} B_{e}
$$

Proof. Assumptions (A1) and (A3) state that $A$ is Schur and the transfer function from $e_{k}$ to $y_{k}$ is minimum-phase. If these are true then, in stationarity, $x_{k}$ can be reconstructed exactly from $\left\{\mathcal{Y}_{k}, \mathcal{I}_{k}, \mathcal{M}\right\}$ via the recursion (26). Implementing the optimal state feedback $i=K x$ gives (69).

In many circumstances it is necessary to balance energy harvesting control objectives against other constraints. A class of sub-optimal energy harvesting controllers can be generated via the certainty-equivalence principle, in the same way they are for standard LQG problems, through the use of a stabilizing Luenberger observer; i.e.,

$$
\begin{aligned}
\chi_{k+1} & =A \chi_{k}+B_{i} i_{k}+L\left(C_{y} \chi_{k}+D_{i y} i_{k}-y_{k}\right) \\
i_{k} & =K \chi_{k}
\end{aligned}
$$


where $L$ is the Luenberger gain, designed by any standard technique, and where $A_{L}=A+L C_{y}$ is asymptotically stable. The resultant performance is then

$$
\bar{p}=\bar{p}_{0}-\operatorname{tr}\left\{K S K^{T} \Delta\right\}
$$

where $S$ is the stationary estimation error covariance; i.e.,

$$
S=A_{L} S A_{L}^{T}+\left(L+B_{e}\right) \Sigma_{e}\left(L+B_{e}\right)^{T}
$$

In this paper we will consider this class of certainty-equivalence controllers, with the understanding that it contains the optimal causal controller; i.e., with $L=-B_{e}$.

\section{Disturbance-adaptive energy harvesting}

In the previous section we determined the optimal causal energy harvesting controller for the situation in which the problem data $\mathcal{M}$ is known with certainty. We now split $\mathcal{M}$ into parameters characterizing the controllable subspace, i.e.,

$$
\mathcal{D}=\left\{A_{11}, B_{i 1}, C_{y 1}, D_{i y}, E_{y q}\right\}
$$

and parameters related only to the disturbance model, i.e.,

$$
\mathcal{N}=\left\{A_{12}, A_{22}, B_{e}, C_{y 2}, \Sigma_{e}\right\}
$$

In this section, we consider the implementation of separation-principle controllers characterized by (70), for the more realistic scenario in which only the parameters in $\mathcal{D}$ are assumed to be known with certainty (e.g., by prior system identification) and the control problem is to design a mapping $\left\{\mathcal{Y}_{k}, \mathcal{I}_{k}, \mathcal{D}\right\} \rightarrow i_{k}$. Our approach here is to estimate $\mathcal{N}$ in real time, from $\left\{\mathcal{Y}_{k}, \mathcal{I}_{k}, \mathcal{D}\right\}$, resulting in an estimate $\hat{\mathcal{N}}_{k}$. We then assume certainty-equivalence and implement the optimal controller $\left\{\mathcal{Y}_{k}, \mathcal{I}_{k},\left\{\mathcal{D}, \hat{\mathcal{N}}_{k}\right\}\right\} \rightarrow i_{k}$, using the results from the previous section. To explain the technique, we assume perfect certainty-equivalence (i.e., that $\hat{\mathcal{N}}_{k}=\mathcal{N}$ ) in Sections 4.1-4.3, before finally substituting $\mathcal{N}$ with $\hat{\mathcal{N}}_{k}$ in Section 4.4. 


\subsection{Isolating disturbance-dependent feedback terms}

We begin by developing a representation of controller (70) which is partitioned into a superposition of two subsystems, $\overline{\mathcal{H}}$ and $\tilde{\mathcal{H}}$. Subsystem $\overline{\mathcal{H}}$ has parameters entirely by determined $\mathcal{D}$, and is therefore deterministic. Subsys-

tem $\tilde{\mathcal{H}}$ has parameters which depend on both $\mathcal{D}$ and $\mathcal{N}$, and must therefore be adapted based on identified values for $\mathcal{N}$.

First, consider the $n_{1}$-state system

$$
\begin{aligned}
& \bar{x}_{k+1}=A_{11} \bar{x}_{k}+B_{i 1} i_{k}+M\left(y_{k}-\bar{y}_{k}\right) \\
& {\left[\begin{array}{l}
\bar{y}_{k} \\
\bar{i}_{k}
\end{array}\right]=\left[\begin{array}{c}
C_{y 1} \\
K_{1}
\end{array}\right] \bar{x}_{k}+\left[\begin{array}{c}
D_{i y} \\
0
\end{array}\right] i_{k}}
\end{aligned}
$$

where $M \in \mathbb{R}^{n_{1} \times n_{y}}$ is an arbitrary matrix such that

$$
A_{M 11} \triangleq A_{11}-M C_{y 1}
$$

is Schur. We note that $\bar{y}$ and $\bar{i}$ is deterministic at time $k$, because they depend only on $\left\{\mathcal{Y}_{k}, \mathcal{I}_{k}, \mathcal{D}\right\}$. Defining

$$
\begin{aligned}
& \tilde{i}_{k}=i_{k}-\bar{i}_{k} \\
& \tilde{y}_{k}=y_{k}-\bar{y}_{k}
\end{aligned}
$$

the above is equivalent to the system we will call $\overline{\mathcal{H}}$, characterized as

$$
\overline{\mathcal{H}}:\left\{\begin{array}{c}
\bar{x}_{k+1}=\left(A_{11}+B_{i 1} K_{1}\right) \bar{x}_{k}+B_{i 1} \tilde{i}_{k}+M \tilde{y}_{k} \\
{\left[\begin{array}{c}
\bar{y}_{k} \\
i_{k}
\end{array}\right]=\left[\begin{array}{c}
C_{y 1}+D_{i y} K_{1} \\
K_{1}
\end{array}\right] \bar{x}_{k}+\left[\begin{array}{c}
D_{i y} \\
I
\end{array}\right] \tilde{i}_{k}}
\end{array}\right.
$$

We then consider the dynamics of the perturbed state vector

$$
\tilde{x}_{k}=x_{k}-E_{1} \bar{x}_{k}
$$

where $E_{1}=\left[\begin{array}{ll}I_{n_{1}} & 0_{n_{1} \times n_{2}}\end{array}\right]^{T}$. Subtracting (75) from (19), we have that the evolution of the perturbed state $\tilde{x}_{k}$ is then governed by

$$
\begin{aligned}
\tilde{x}_{k+1} & =A_{M} \tilde{x}_{k}+B_{M} e_{k} \\
\tilde{y}_{k} & =C_{y} \tilde{x}_{k}+e_{k}
\end{aligned}
$$


where

$$
\begin{aligned}
& A_{M}=A-E_{1} M C_{y}=\left[\begin{array}{cc}
A_{M 11} & A_{M 12} \\
0 & A_{22}
\end{array}\right] \\
& B_{M}=B_{e}-E_{1} M
\end{aligned}
$$

Note that $A_{M 11}$ is as in (76), while $A_{M 12}=A_{12}-M C_{y 2}$. Because $A_{M 11}$ is Schur, this implies that $A_{M}$ is as well. Consequently, the stochastic response of (81) is stationary. Because $\tilde{x}_{k}$ is uncertain, it must be estimated via an observer, and then $\tilde{i}_{k}$ determined via certainty-equivalence. Thus we have the the part of the controller depending on the uncertain parameters $\mathcal{N}$, which we will call $\tilde{\mathcal{H}}$, characterized by

$$
\tilde{\mathcal{H}}:\left\{\begin{array}{l}
\tilde{\chi}_{k+1}=\left(A_{M}+L_{M} C_{y}\right) \tilde{\chi}_{k}-L_{M} \tilde{y}_{k} \\
\tilde{i}_{k}=K \tilde{\chi}_{k}
\end{array}\right.
$$

where $L_{M}$ is the Luenberger gain for the observer.

It is straight-forward to show that with the observer gains related via

$$
L_{M}=L+E_{1} M
$$

the controller state $\chi_{k}$ from (70) is equal to

$$
\chi_{k}=\tilde{\chi}_{k}+E_{1} \bar{x}_{k}
$$

As such, combining $\overline{\mathcal{H}}$ and $\tilde{\mathcal{H}}$ as in Figure 3, we have an equivalent realization to controller (70).

\subsection{Control design}

Knowledge of $\tilde{\mathcal{H}}$ requires the knowledge of $\mathcal{N}$, which we presume to be uncertain, and which must be estimated from the system response. Thus, the

controller indirectly adapts $\tilde{H}$ by estimating $\mathcal{N}$ and then determining $\tilde{\mathcal{H}}$ from this estimate assuming certainty-equivalence. This adaptation is accomplished by recognizing that, since $\tilde{y}_{k}$ is available for feedback, $\mathcal{N}$ can be estimated by output-only stochastic system identification of the system in (81). 


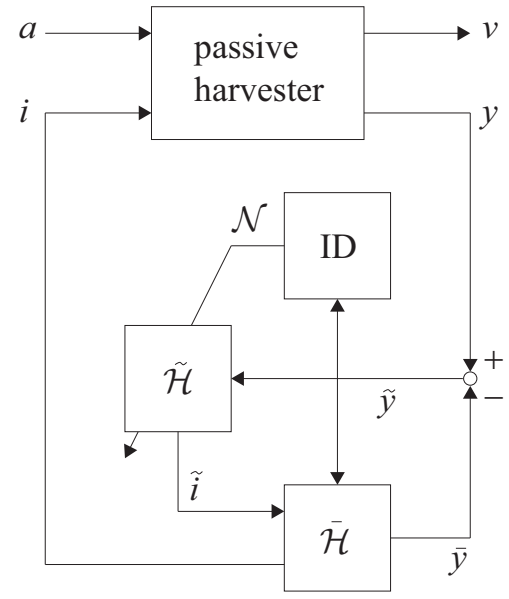

Figure 3: Block diagram of feedback partitioning

In the estimation of $\mathcal{N}$, any basis for $x_{2}$ is equivalent. To eliminate the ambiguity related to the basis of the identified parameters in $\mathcal{N}$, it will be useful to refer to the transfer function representation of the mapping $\tilde{\mathcal{H}}: \tilde{y} \rightarrow \tilde{i}$ directly in terms of its Markov parameters, which we denote $\left\{\tilde{H}_{1}, \tilde{H}_{2}, \ldots\right\}$; i.e.,

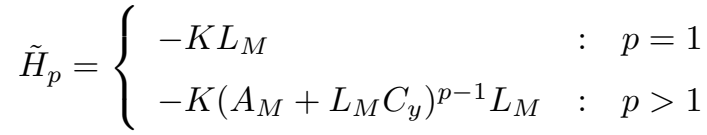

We then have that $\tilde{\mathcal{H}}$ is equivalently represented by the convolution

$$
\tilde{i}_{k}=\sum_{p=1}^{\infty} \tilde{H}_{p} \tilde{y}_{k-p}
$$

Our approach here will be to approximate this convolution by truncating the summation to the first $N$ terms, thus creating a FIR filter subsystem to determine $\tilde{i}_{k}$.

Alternatively, one could implement $\tilde{\mathcal{H}}$ exactly, by relating its state space parameters to an associated ARMA model

$$
\tilde{i}_{p}=\sum_{p=1}^{n}\left(-\tilde{D}_{p} \tilde{i}_{k-p}+\tilde{N}_{p} \tilde{y}_{k-p}\right)
$$

via a coprime factorization. This approach was previous implemented by the authors (Nie \& Scruggs, 2014). The problem with this is that it creates a 
"hidden" feedback loop, in applications where the $\tilde{D}_{p}$ and $\tilde{N}_{p}$ parameters are re-identified at each time step. Specifically, time-varying fluctuations in these parameters propagate into the dynamics of $\tilde{i}$, due to the autoregressive terms above. In the example application considered here, this effect was highly significant, resulting in instabilities and "bursting" phenomena, unless the rate of parametric adaptation was severely restricted.

We thus arrive at the final realization of controller (70), as

$$
\begin{aligned}
\bar{x}_{k+1} & =\left(A_{11}+B_{i 1} K_{1}\right) \bar{x}_{k}+B_{i 1} \tilde{i}_{k}+M \tilde{y}_{k} \\
i_{k} & =K_{1} \bar{x}_{k}+\tilde{i}_{k} \\
\tilde{y}_{k} & =y_{k}-\left(C_{y 1}+D_{i y} K_{1}\right) \bar{x}_{k}-D_{i y} \tilde{i}_{k} \\
\tilde{i}_{k} & =\sum_{p=1}^{N} \tilde{H}_{p} \tilde{y}_{k-\ell}
\end{aligned}
$$

Given $\{\mathcal{D}, \mathcal{N}\}$, the design of this controller thus comes down to determining $M$, $L_{M}$, and the FIR truncation length $N$. A variety of techniques can be used to determine these parameters. Below, we describe techniques that were used in the examples at the end of the paper.

- Determining $M$ : For the case where $\mathcal{N}$ is known, $M$ is transparent in the implementation of the controller; i.e., it has no influence on the closedloop dynamics, so long as $L_{M}$ is also varied to produce the same $L$ in (85). However, $M$ is useful because it modifies the dynamics of the innovations model (81), which is the stochastic system from which the parameters $\mathcal{N}$ are identified. Recalling that $A_{M 11}$ is determined solely by the deterministic parameters $\mathcal{D}$ (together with $M$ ), we see that by choosing $M$ appropriately, the dynamics of $A_{M 11}$ can be affected in such a way as to make it easier to identify the unknown parameters in the system; i.e., $A_{12}$ (or equivalently $A_{M 12}$ ), $A_{22}, C_{2}, B_{e}$, and $\Sigma_{e}$.

In this paper, we assume $M=B_{i 1} M^{\prime}$, where $M^{\prime} \in \mathbb{R}^{n_{p} \times n_{p}}$ is optimized to minimize the maximum modulus of the eigenvalues of $A_{M 11}$. However, this is admittedly an ad-hoc choice for $M$, and other design choices could 
justifiably be made.

- Determining $L_{M}$ : We take $L_{M}$ to be the Kalman gain for a feedback system as described above, but with supplemental fictitious white noise of intensity $\Sigma_{f} \geqslant 0$ injected into the feedback loop. Thus, we have that $L$ is solved via the Riccati equation

$$
\begin{aligned}
S=A_{M} S A_{M}^{T}+ & B_{M} \Sigma_{e} B_{M}^{T}-\left(A_{M} S C_{y}^{T}+B_{M} \Sigma_{e}\right) \\
& \times\left(\Sigma_{e}+\Sigma_{f}+C_{y} S C_{y}^{T}\right)^{-1}\left(A_{M} S C_{y}^{T}+B_{M} \Sigma_{e}\right)^{T}
\end{aligned}
$$

which is guaranteed to have a unique stabilizing solution if $A_{M}$ is Schur. In order to systematize the controller design technique, our convention will be to choose $\Sigma_{f}$ to be such that the fictitious noise would have a RMS noise-to-signal ratio of $\alpha$; i.e., we take

$$
\Sigma_{f}=\alpha^{2} \times\left(C_{y} X C_{y}^{T}+\Sigma_{e}\right)
$$

where $X=X^{T}=\mathcal{E} \tilde{x} \tilde{x}^{T}$ is the solution to

$$
X=A_{M} X A_{M}^{T}+B_{M} \Sigma_{e} B_{M}^{T}
$$

As $\alpha \rightarrow 0, L_{M} \rightarrow-B_{M}$ and $S \rightarrow 0$, provided that $e \mapsto \tilde{y}$ is minimumphase. However, this is guaranteed. To see this, consider that the transfer function from $e$ to $\tilde{y}$ can be expressed as

$$
G_{e \tilde{y}}(z)=\left[I+C_{y 1}\left(z I-A_{11}\right)^{-1} M\right]^{-1} U(z)
$$

The first transfer function in the product above is guaranteed to be stable if $A_{M 11}$ is Schur, and its inverse is also guaranteed to be stable since $A_{11}$ is Schur. Consequently, it is minimum-phase. Meanwhile, the second transfer function is guaranteed to be minimum-phase by Assumption (A3). For the case where $\mathcal{N}$ is known precisely, it thus follows from (85) and the above observations that $L \rightarrow-B_{e}$ as $\alpha \rightarrow 0$, and consequently the conroller in (90) asymptotically approaches the causal optimal controller (69) as $\alpha \rightarrow 0$ and $N \rightarrow \infty$. 
However, a finite value of $\alpha$ is beneficial in practice, to ameliorate numerical difficulties encountered in the implementation of the identification algorithm for $\mathcal{N}$. For the example considered in this paper, $\alpha=0.05$ was used.

- Determining $N$ : Clearly, $N$ should be chosen to be large enought that the Markov parameters $\tilde{H}_{p} \approx 0$ for $p>N$. The minimum value of $N$ which achieves this is dependent on the choices of $M$ and $L_{M}$. However, for the example considered in this paper, $N$ was not explicitly optimized, and was instead chosen to be conservatively large. While there might be some slight computational advantages of minimizing $N$, these were not deemed of high concern for the target application of wave energy conversion, due to the low sample rate being considered $(T=1 \mathrm{~s})$. It was found that setting $N=250$ was more than sufficient for all sea states considered.

\subsection{Stochastic identification of $\mathcal{N}$}

This subsection overviews the determination of $\mathcal{N}$, assuming certainty of $\mathcal{D}$, and assuming adquate response data to accurately evaluate expectations from time averages. Specifically, we are concerned with the autocorrelation function for the innovations process (81); i.e.,

$$
R_{p} \triangleq \mathcal{E} \tilde{y}_{k+p} \tilde{y}_{k}^{T}, p \in \mathbb{Z}_{\geqslant 0}
$$

Subspace-based system identification techniques are used (Katayama, 2005). The primary justification for this is that subspace-based techniques scale well to large system models, and to systems with many transducers. Within the subspace-based paradigm, our objective can be accomplished by any of several related algorithms, most of which are variants of the methods of Faurre (1976) or Akaike (1975). Akaike-based methods were chosen here, for the distinct advantage that they avoid the need to solve a passivity-type Riccati equation as part of the identification procedure. As is well known, this Riccati equation may fail to have a solution in practice, due to errors in estimation of $R_{p}$. Although the Faurre-based approach can be augmented to be robust against such failures 
(see, e.g., Mari et al. (2000)), the Akaike-based methodology discussed here is more straight-forward.

In stationarity, the state covariance matrix $X=\mathcal{E} \tilde{x} \tilde{x}^{T}$ is the solution to (93), and the coefficients $\left\{R_{p}, p \in \mathbb{Z}_{\geqslant 0}\right\}$ are related to the state space parameters in (81) via

$$
R_{p} \triangleq R_{y 0}(p T)= \begin{cases}C_{y} X C_{y}^{T}+\Sigma_{e} & : p=0 \\ C_{y} A_{M}^{p} X C_{y}^{T} & : p>0\end{cases}
$$

Following the standard Akaike algorithm for stochastic realization, define the family of autocorrelation matrices $P_{\ell}$ as

$$
P_{\ell}=\left[\begin{array}{cccc}
R_{0} & R_{1} & \cdots & R_{\ell-1} \\
R_{1}^{T} & R_{0} & & R_{\ell-2} \\
\vdots & & \ddots & \vdots \\
R_{\ell-1}^{T} & & \cdots & R_{0}
\end{array}\right]
$$

and the family of Hankel matrices $Q_{\ell, m}$ as

$$
Q_{\ell, m}=\left[\begin{array}{cccc}
R_{1} & R_{2} & \cdots & R_{m} \\
R_{2} & R_{3} & \cdots & R_{m+1} \\
\vdots & \vdots & \ddots & \vdots \\
R_{\ell} & R_{\ell+1} & \cdots & R_{\ell+m-1}
\end{array}\right]
$$

We also associate with $P_{\ell}$ the family of factorizations $\Gamma_{\ell}$, such that

$$
P_{\ell}=\Gamma_{\ell} \Gamma_{\ell}^{T}
$$

where we note that although any such factorization is valid for the purposes of the algorithm to be presented, we uniformly assume an upper Cholesky factorization. We further define the canonical correlation matrix as

$$
\Xi=\Gamma_{\ell}^{-1} Q_{\ell, m} \Gamma_{m}^{-T}
$$

For $\ell, m>n$, we also define the extended observability and controllability 
matrices as

$$
\mathcal{O}_{\ell}=\left[\begin{array}{c}
C_{y} \\
C_{y} A_{M} \\
\vdots \\
C_{y} A_{M}^{\ell-1}
\end{array}\right] \quad \mathcal{C}_{m}=\left[\begin{array}{c}
\bar{C} \\
\bar{C} A_{M}^{T} \\
\vdots \\
\bar{C}\left(A_{M}^{T}\right)^{m-1}
\end{array}\right]^{T}
$$

where $\bar{C}=C_{y} X A_{M}^{T}$, and where we note that $Q_{\ell, m}=\mathcal{O}_{\ell} \mathcal{C}_{m}$. Given $\mathcal{O}_{\ell}$ and $\mathcal{C}_{m}$, $A_{M}, C_{y}$, and $\bar{C}$ can be inferred from

$$
\begin{aligned}
A_{M} & =\underline{\mathcal{O}}_{\ell}^{\dagger} \overline{\mathcal{O}}_{\ell} \\
C_{y} & =\mathcal{O}_{\ell}\left(1: n_{y}, 1: n\right) \\
\bar{C} & =\mathcal{C}_{m}\left(1: n, 1: n_{y}\right)^{T}
\end{aligned}
$$

where $\underline{\mathcal{O}}_{\ell}$ and $\overline{\mathcal{O}}_{\ell}$ in (102) denote the removal of the last $n_{y}$ rows, and first $n_{y}$ rows, respectively, and the parentheses in (103) and (104) denote row and column ranges. We then have the following theorem (see, e.g., Katayama (2005), Theorem 8.4).

Theorem 2. For any $\ell, m>n$, evaluate the singular value decomposition of $\Xi=U \Sigma V^{T}$ where $\Sigma$ is diagonal and positive definite. Then $\Sigma \in \mathbb{R}^{n \times n}$ and all the minimal stochastic realizations of (81) are related through a similarity transformation matrix $T$, with $X=T \Sigma^{2} T^{T}$, and in which $\mathcal{O}_{\ell}$ and $\mathcal{C}_{m}$ in (101) are

$$
\begin{gathered}
\mathcal{O}_{\ell}=\Gamma_{\ell} U T^{-1} \\
\mathcal{C}_{m}=T \Sigma V^{T} \Gamma_{m}^{T}
\end{gathered}
$$

It follows that $A_{M}, C_{y}$, and $\bar{C}$ are related through (102), (103), and (104), and

$$
\begin{aligned}
\Sigma_{e} & =R_{0}-C_{y} X C_{y}^{T} \\
B_{M} & =\left(\bar{C}^{T}-A X C_{y}^{T}\right) \Sigma_{e}^{-1}
\end{aligned}
$$

The system identification procedure suggested by Theorem 2 could be applied as presented, with arbitrary invertible $T \in \mathbb{R}^{n \times n}$, resulting in a realization 
for (81). However, we require a realization which preserves the basis for the controllable subspace $x_{1}$, and our identified $A_{M}$ should have the deterministic values for $A_{M 11}$ and $C_{y 1}$. We must also protect against the circumstance in which a subspace of $x_{1}$ is uncontrollable from $e$, but are controllable from $i$. In this circumstance, the above procedure will find a realization of order less than $n$. We must therefore modify the procedures above.

Define the extended observability matrix for the controllable subspace $x_{1}$ as

$$
\mathcal{O}_{\ell 1}=\left[\begin{array}{c}
C_{1} \\
C_{1} A_{M 11} \\
\vdots \\
C_{1} A_{M 11}^{\ell-1}
\end{array}\right]
$$

and note that this matrix is deterministic. Define the associated unitary matrix $\bar{U}$ as

$$
\bar{U}=\Gamma_{\ell}^{-1} \mathcal{O}_{\ell 1}\left(\mathcal{O}_{\ell 1}^{T} P_{\ell}^{-1} \mathcal{O}_{\ell 1}\right)^{-1 / 2}
$$

Next, define the canonical correlation matrix $\tilde{\Xi}$ as the matrix in (100) with the range space associated with $\bar{U}$ removed; i.e.,

$$
\tilde{\Xi}=\left(I-\bar{U} \bar{U}^{T}\right) \Gamma_{\ell}^{-1} Q_{\ell, m} \Gamma_{m}^{-T}
$$

and evaluate the singular value decomposition $\tilde{\Xi}=\tilde{U} \tilde{\Sigma} \tilde{V}^{T}$, with the convention that $\tilde{\Sigma}$ is diagonal and positive-definite. It then follows that

$$
\Xi=\left[\begin{array}{ll}
\bar{U} & \tilde{U}
\end{array}\right]\left[\begin{array}{c}
\bar{U}^{T} \Xi \\
\tilde{\Sigma} \tilde{V}^{T}
\end{array}\right]
$$

Now, conduct a second singular value decomposition

$$
\left[\begin{array}{c}
\bar{U}^{T} \Xi \\
\Sigma_{22} V_{2}^{T}
\end{array}\right]=Y \Sigma V^{T}
$$

where we use the convention that $Y$ is square and unitary, while $\Sigma$ is diagonal and positive semidefinite. Then we now have the decomposition $\Xi=U \Sigma V^{T}$ as in Theorem 2, but with

$$
U=\left[\begin{array}{cc}
\bar{U} & \tilde{U}
\end{array}\right] Y
$$


and with $\Sigma$ positive semidefinite (but not necessarily definite). If $\Sigma$ fails to be positive definite, this implies that $x_{1}$ has a subspace that is uncontrollable from e.

Now, to determine a similarity transformation matrix $T$, we require that for $\mathcal{O}_{\ell}$ evaluated as in (105), $T$ must be such that

$$
\begin{aligned}
\underline{\mathcal{O}}_{\ell}^{\dagger} \overline{\mathcal{O}}_{\ell} E_{1} & =\left[\begin{array}{c}
A_{M 11} \\
0
\end{array}\right] \\
\mathcal{O}_{\ell}\left(1: n_{y}, 1: n_{1}\right) & =C_{y 1}
\end{aligned}
$$

But, recalling the definition of $\bar{U}$, we have that

$$
\begin{aligned}
\mathcal{O}_{\ell} & =\left[\begin{array}{ll}
\mathcal{O}_{\ell 1}\left(\mathcal{O}_{\ell 1}^{T} P_{\ell}^{-1} \mathcal{O}_{\ell 1}\right)^{-1 / 2} & \Gamma_{\ell} \tilde{U}
\end{array}\right] Y T^{-1} \\
& =\left[\begin{array}{ll}
\mathcal{O}_{\ell 1} & \Gamma_{\ell} \tilde{U}
\end{array}\right]\left[\begin{array}{cr}
\left(\mathcal{O}_{\ell 1}^{T} P_{\ell}^{-1} \mathcal{O}_{\ell 1}\right)^{-1 / 2} & 0 \\
0 & I
\end{array}\right] Y T^{-1}
\end{aligned}
$$

We see that any similarity transformation matrix of the form

$$
T=\left[\begin{array}{ll}
I & \Psi_{12} \\
0 & \Psi_{22}
\end{array}\right]\left[\begin{array}{cc}
\left(\mathcal{O}_{\ell 1}^{T} P_{\ell}^{-1} \mathcal{O}_{\ell 1}\right)^{-1 / 2} & 0 \\
0 & I
\end{array}\right] Y
$$

where $\Psi_{12}$ and $\Psi_{22}$ are arbitrary real matrices with $\Psi_{22}$ invertible, gives the required values for $A_{11}$ and $C_{1}$. It turns out that the above transformation also results in $A_{21}=0$. To see this, let $\Psi=\left[\begin{array}{ll}\Psi_{12}^{T} & \Psi_{22}^{T}\end{array}\right]^{T}$ and consider that assuming the above for $T, A_{M}$ is the solution to the least squares problem

$$
\begin{aligned}
A_{M}= & \underset{\Upsilon}{\operatorname{argmin}}\left\|\left[\begin{array}{ll}
\underline{\mathcal{O}}_{\ell 1} & \underline{\Gamma}_{\ell} \tilde{U} \Psi
\end{array}\right] \Upsilon-\left[\begin{array}{ll}
\overline{\mathcal{O}}_{\ell 1} & \bar{\Gamma}_{\ell} \tilde{U} \Psi
\end{array}\right]\right\|_{F}^{2} \\
= & \underset{\Upsilon}{\operatorname{argmin}}\left\|\left[\begin{array}{ll}
\underline{\mathcal{O}}_{\ell 1} & \underline{\Gamma}_{\ell} \tilde{U} \Psi
\end{array}\right] \Upsilon-\left[\begin{array}{ll}
\underline{\mathcal{O}}_{\ell 1} A_{M 11} & \bar{\Gamma}_{\ell} \tilde{U} \Psi
\end{array}\right]\right\|_{F}^{2} \\
= & \underset{\Upsilon_{11}, \Upsilon_{21}}{\operatorname{argmin}}\left\|\underline{\mathcal{O}}_{\ell 1}\left(\Upsilon_{11}-A_{M 11}\right)+\underline{\Gamma}_{\ell} \tilde{U} \Psi \Upsilon_{21}\right\|_{F}^{2} \\
& +\underset{\Upsilon_{12}, \Upsilon_{22}}{\operatorname{argmin}}\left\|\left[\begin{array}{ll}
\underline{\mathcal{O}}_{\ell 1} & \underline{\Gamma}_{\ell} \tilde{U} \Psi
\end{array}\right]\left[\begin{array}{l}
\Upsilon_{12} \\
\Upsilon_{22}
\end{array}\right]-\bar{\Gamma}_{\ell} \tilde{U} \Psi\right\|_{F}^{2}
\end{aligned}
$$

We can see clearly that the solution to the first of the two minimizations will always result in $\Upsilon_{11}=A_{M 11}$ and $\Upsilon_{21}=0$. As such, we may choose $\Psi_{12}$ and $\Psi_{22}$ arbitrarily, and the most obvious choice is 0 and $I$, respectively. 
We therefore have an algorithm for identification of $\mathcal{N}$. The algorithm proceeds, given correlation data $\left\{R_{p}, p \in \mathbb{Z}_{\geqslant 0}\right\}$, and integers $\ell, m>n$, by the following steps:

1. Assemble $P_{\ell}, P_{m}$, and $Q_{\ell, m}$, and evaluate the Cholesky factorizations $\Gamma_{\ell}$ and $\Gamma_{m}$.

2. Find $\Xi$ and $\bar{U}$, and from these, $\tilde{\Xi}$.

3. Evaluate the singular value decomposition $\tilde{\Xi}=\tilde{U} \tilde{\Sigma} \tilde{V}^{T}$.

4. Evaluate $X=T \Sigma^{2} T^{T}$. Note that rather than doing another singular value decomposition, this can be found equivalently as

$$
X=\left[\begin{array}{cc}
\Lambda & 0 \\
0 & I
\end{array}\right]\left[\begin{array}{c}
\bar{U}^{T} \\
\tilde{U}^{T}
\end{array}\right] \Xi \Xi^{T}\left[\begin{array}{ll}
\bar{U} & \tilde{U}
\end{array}\right]\left[\begin{array}{cc}
\Lambda & 0 \\
0 & I
\end{array}\right]
$$

where $\Lambda=\left(\mathcal{O}_{\ell 1}^{T} P_{\ell}^{-1} \mathcal{O}_{\ell 1}\right)^{-1 / 2}$.

5. Evaluate $\mathcal{O}_{\ell}$ and $\mathcal{C}_{m}$, which may be conveniently found as

$$
\begin{aligned}
\mathcal{O}_{\ell} & =\left[\begin{array}{ll}
\mathcal{O}_{\ell 1} & \Gamma_{\ell} \tilde{U}
\end{array}\right] \\
\mathcal{C}_{m} & =\left[\begin{array}{ll}
\Lambda & 0 \\
0 & I
\end{array}\right]\left[\begin{array}{l}
\bar{U}^{T} \\
\tilde{U}^{T}
\end{array}\right] \Xi
\end{aligned}
$$

6. Find $C_{y 2}, A_{M 12}, A_{22}, B_{M}$, and $\Sigma_{e}$ as in Theorem 2.

We note that this algorithm is not much more computationally expensive than the standard algorithm from Theorem 2, because both algorithms must evaluate the Cholesky factorizations, and both algorithms contain only one singular value decomposition.

\subsection{Recursive identification of $\mathcal{N}$ from data}

The adaptive controller described in (90) is implemented at each time $k$, using the Markov parameters $\left\{\tilde{H}_{p}: p \in\{1 \ldots N\}\right\}$ resulting from the present estimate of $\mathcal{N}$, denoted

$$
\hat{\mathcal{N}}_{k}=\left\{\hat{A}_{12}(k), \hat{A}_{22}(k), \hat{C}_{y 2}(k), \hat{B}_{e}(k), \hat{\Sigma}_{e}(k)\right\}
$$


Each term in the sequence $\hat{\mathcal{N}}_{k}$ is estimated using the methodology described at the end of Section 4.3, using the prior estimate of $\left\{R_{p}: p \in\{0, . . \ell+m-1\}\right\}$, which we denote $\left\{\hat{R}_{p}(k): p \in\{0, \ldots \ell+m-1\}\right\}$. To estimate each $\hat{R}_{p}(k)$, one faces a fundamental tradeoff. If it is desired to make the controller quick to adapt, $\hat{R}_{p}(k)$ should be made using only recent values of $\tilde{y}$. However, if it is desired to make the estimate accurate, then one should use as much data as possible, to minimize the variance of the estimate.

For the purposes of this paper, we assume that $\hat{R}_{p}(k)$ is obtained through a standard first-order auto-regressive averaging filter; i.e.,

$$
\hat{R}_{p}(k)=\beta \hat{R}_{p}(k-1)+(1-\beta) \tilde{y}_{k-1} \tilde{y}_{k-1-\ell}^{T}
$$

where $\beta \in(0,1)$ is the forgetting factor. Rather than specifying $\beta$ directly, we characterize it by the half life $N_{\beta}$ associated with the averaging filter, equal to

$$
N_{\beta}=\ln (1 / 2) / \ln \beta
$$

It must of course be the case that $N_{\beta}$ should be much larger than the characteristic time constants associated with the controller. This means that $N_{\beta} \gg N$, and also that $N_{\beta} \gg \max \{\ell, m\}$ (i.e., the dimensions of the Hankel matrix $Q_{\ell, m}$ ). However, $N_{\beta}$ should also be commensurate with the time durations over which $\mathcal{N}$ can be expected to vary. In application to wave energy conversion, we expect $\mathcal{N}$ to vary due to changes in the weather and tide, as well as other factors that can be expected to change on an hourly time scale. Consequently, at a sample time of $T=1 \mathrm{~s}$, this imples that $N_{\beta}=3600$ is appropriate.

The algorithm described in Section 4.3 is robust to perturbations in $R_{p}$, due to estimation error, and will generally execute even if the estimates are very poor. (Although it is of course the case that performance of the controller will suffer.) Nonetheless, there are a few augmentations to the general approach described in previous subsections which result in superior performance and reliability.

- It is not necessarily the case that the accuracy of the identification algorithm increases as $\ell$ and $m$ are increased, because an extremely long series 
of ouptuts are necessary to get accurate estimates for $R_{p}$ for $p$ large. Consequently, for a given forgetting factor $\beta$, there will in general be finite values of $\ell$ and $m$ above which the identification algorithm can behave erratically. For the choice made here (i.e., $N_{\beta}=3600$ ), and given the dimension of the state space to be identified was $n=11, \ell=m=50$ was found to be appropriate.

- For stochastic disturbances with high quality factor, estimation errors in $\hat{R}_{\ell}(k)$ may result in an estimation $\hat{A}_{22}(k)$ which is not Schur. Technically, this occurrence will not result in failure of the controller, because even if $\hat{A}_{22}(k)$ is unstable, a Kalman gain $L_{M}$ will still in general exist, and if so, it will stabilize $\tilde{\mathcal{H}}$. Nonetheless, loss of stability of $\hat{A}_{22}(k)$ tends to hamper performance. To guard against this, we resort to the common ad-hoc practice of checking the eigenvalues of $\hat{A}(k)$ at each iteration, and radially reflecting any unstable eigenvalues into the unit circle. In other words, if $\lambda$ is an unstable eigenvalue, it is replaced in the eigenvalue decomposition with $\lambda /|\lambda|^{2}$.

- The assumption of certainty equivalence loses validity if the FIR coefficients $\tilde{H}_{\ell}$ are functions of the same values of $\tilde{y}_{k}$ by which they multiply in controller (90). To address this, a hindcast time delay the same length of the FIR filter (i.e., $N$ ) was imposed on the estimate $\hat{R}_{p}(k)$. In other words, (127) was modified to

$$
\hat{R}_{p}(k)=\beta \hat{R}_{p}(k-1)+(1-\beta) \tilde{y}_{k-1-N} \tilde{y}_{k-1-N-p}^{T}
$$

- The fictitious noise term used in the design of the observer gain $L_{M}$ for iteration $k$ is $\Sigma_{f}=\alpha^{2} \hat{R}_{0}(k)$, where $\alpha$ is the noise-to-signal ratio. There are two computational steps conducted each time $\tilde{H}$ is adapted, which can fail due to numerical inaccuracy, which can be addressed through the inclusion of $\Sigma_{f}$. The first of these is the evaluation of the Cholesky factorizations for $P_{\ell}$ and $P_{m}$, which will fail if these matrices fail to be positive 
definite. Theoretically they should always be so, even if the $\hat{R}_{p}(k)$ estimates are inaccurate, but in practice this may not be the case due to numerical error. This issue can be averted by adding the supplemental noise to the diagonals of these matrices, thus guaranteeing minimal eigenvalues of $\Sigma_{f}$. The second point of potential failure is in the evaluation of Riccati equation (91), which may occur if the estimate $\hat{\Sigma}_{e}(k)$ is very small. This issue can is averted by the inclusion of the supplemental noise term $\Sigma_{f}$ in the Riccati equation, as originially described. However, if $\Sigma_{f}$ is used to ameliorate both the numerical issues above, then it has been "added twice" in the analysis, which is then effectively conducted with a supplemental noise term of $\Sigma_{f}=\left(\left(1+\alpha^{2}\right)^{2}-1\right) \hat{R}_{0}(k)$. To accommodate this, we merely change our interpretation of $\alpha$ such that the desired noiseto-signal ratio is equal to $\sqrt{2 \alpha^{2}+\alpha^{4}}$. So for exmple, for a desired ratio of 0.05 , one would set $\alpha \approx 0.035$.

- The requirement to solve singular value decomposition for the identification algorithm, at each time step, becomes computationally intensive as the Hankel dimensions $\{\ell, m\}$ are made large. For the present problem, this was not deemed a great concern because acceptable performance was achieved with $\ell=m=50$, and at that size the algorithm is extremely efficient. However, if the given application required larger Hankel dimensions, or a much shorter sample time, the true singular value decomposition could be replaced by an approximate solution, using a subspace tracker, such as the algorithm proposed by Goethals et al. (2004). The authors tested this particular subspace tracking algorithm on the example application, with reasonable (but not quite as favorable) results. However, because it would not be warranted for the target application, this algorithm was not implemented in results shown for the example in the following section. 


\section{Example}

Consider the floating cylindrical buoy in Figure 4. This system constitutes a very simple example of an ocean wave energy harvester with an electric machine as a power take-off mechanism (Mueller, 2002; Polinder et al., 2004; Leijon et al., 2005). The buoy is interfaced with a power generator through a pre-tensioned cable. We assume the tension for this cable is supplied by a constant-force spring, resulting in the tension being equal to $t=t_{0}+\kappa i$ where $t_{0}$ is the tension set point supplied by the constant-force spring, and $\kappa$ is the coupling factor relating the modulation in the tension force to the generator current. The disturbance $a$ is taken to be the incident wave elevation at the buoy. Transfer functions $Z_{a v}(j \omega)$ and $Z_{i v}(j \omega)$ are characterized in the frequency domain as

$$
\begin{aligned}
& Z_{i v}(j \omega)=\frac{\kappa^{2} j \omega}{k+c(\omega) j \omega-m(\omega) \omega^{2}}+r \\
& Z_{a v}(j \omega)=\frac{\kappa f_{a}(j \omega) j \omega}{k+c(\omega) j \omega-m(\omega) \omega^{2}}
\end{aligned}
$$

Static variables $k$ and $r$ are, respectively, the hydrostatic stiffness and the coil resistance of the generator. Frequency-dependent parameters $f_{a}(j \omega), c(\omega)$, and $m(\omega)$ are, respectively, the hydrodynamic force transfer function for the incident wave, the radiation damping, and the added mass. These terms must be solved numerically at each frequency by first solving the associated partial differential equations characterizing the flow field around the buoy, and then integrating the resultant pressure field around the wetted area. For the situation with the buoy restrained at heave elevation $z(t)=z_{0}$ (i.e., its static value) and $a(t)=e^{j \omega t}$, this integral gives the excitation force $f_{a}(j \omega)$. For the situation with an oscillating buoy displacement $z(t)=z_{0}+e^{j \omega t}$ and $a(t)=0$, this integral gives a complex value of $f_{h}(j \omega)=k+c(\omega) j \omega-m(\omega) \omega^{2}$. Because the hydrodynamic stiffness $k$ can be determined from the geometry of the buoy, the terms $c(\omega)$ and $m(\omega)$ can be obtained uniquely from this force.

Numerical solutions for $f_{a}(j \omega), c(\omega)$ and $m(\omega)$ are shown in Figure 5. The fact that they are solutions to partial differential equations implies that the system is infinite-dimensional. We refer the reader to (Scruggs et al., 2013) 


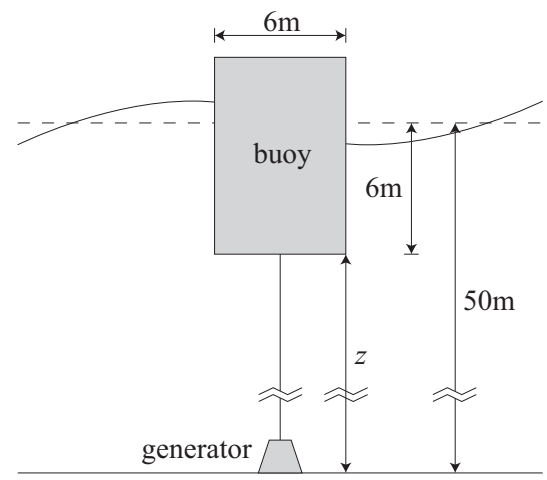

Figure 4: Wave energy harvesting buoy

for more information on how these parameters are calculated from the system geometry and frequency $\omega$.

The stochastic dynamics associated with wave elevation are typically modeled as a stationary stochastic process, with an assumed power spectral density $S_{a}(\omega)$. The most prevalent parametrization of $S_{a}(\omega)$ is the JONSWAP spectrum Faltinsen (1990). This spectrum is parametrized by its mean wave period $T_{1}$, significant wave height $H_{1 / 3}$ (i.e., the mean value of the top third of the distribution of peak-to-trough wave heights), and sharpness factor $\gamma$. The sharpness factor $\gamma$ is constrained to be between 1 and 3.3, the former describing a fully developed sea state and the latter providing a spectrum with a higher quality factor. In terms of these parameters, $S_{a}$ is

$$
S_{a}(\omega)=155 \pi \frac{H_{1 / 3}^{2}}{T_{1}^{4} \omega^{5}} \exp \left[\frac{-944}{T_{1}^{4} \omega^{4}}\right] \gamma^{\nu},
$$

where

$$
\nu=\exp \left[-\left(\frac{0.191 \omega T_{1}-1}{\sqrt{2} \phi}\right)^{2}\right], \quad \phi=\left\{\begin{array}{cc}
0.07 & : \omega T_{1} \leq 5.24 \\
0.09 & : \omega T_{1}>5.24
\end{array}\right.
$$

Clearly, the above spectrum does not constitute a rational function of $\omega^{2}$, and thus is not the result of a stationary, finite-dimensional Gauss-Markov process. Rather, it is the spectrum of an infinite-dimensional stochastic system. ${ }^{1}$ As-

\footnotetext{
${ }^{1}$ We recall that our convention for $S_{a}(\omega)$ follows the control literature; i.e., $\mathcal{E} a^{2}=$
} 


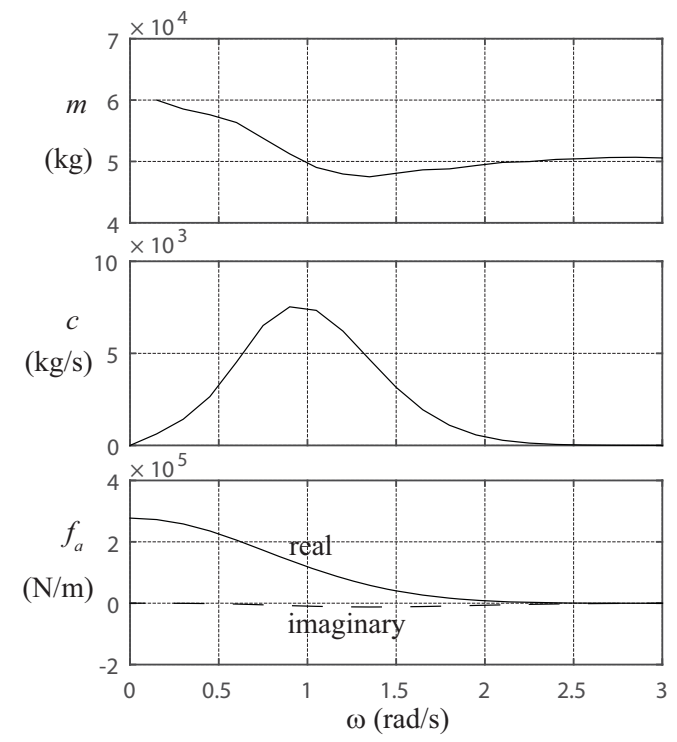

Figure 5: Hydrodynamic coefficients for buoy in Figure 4

sociated with a sea state is the incident wave power flux (i.e., incident power per meter of wave crest), which we denote $p_{I}$ (Falnes, 2002). For stationary stochastic waves, and keeping in mind our normalization convention for $S_{a}(\omega)$, this is

$$
p_{I}=\frac{1}{\pi} \int_{0}^{\infty} \frac{\rho g \omega}{2 k(\omega)}\left(1+\frac{2 k(\omega) h}{\sinh (2 k(\omega) h)}\right) S_{a}(\omega) d \omega
$$

where $\rho$ is the density of water, $g$ is gravitational acceleration, $h$ is the water depth, and $k(\omega)$ is the wave number at frequency $\omega$; i.e., the real solution to the dispersion relation $\omega=\sqrt{(g k) \tanh (k h)}$.

Electrical parameters $\kappa$ and $r$ may be specified individually, but it can be shown that only one combination of them, $c_{e}=\kappa^{2} / r$, influences power generation. This quantity, which has units of viscous damping, represents the effective viscous damping that would be imposed on the system by the transducer with its coils shorted. Here, we assume $c_{e}=10^{6} \mathrm{~kg} / \mathrm{s}$.

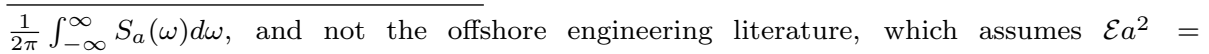
$\int_{0}^{\infty} S_{a}(\omega) d \omega$. This accounts for the discrepancy between (132) and expressions for the JONSWAP spectrum found elsewhere. 


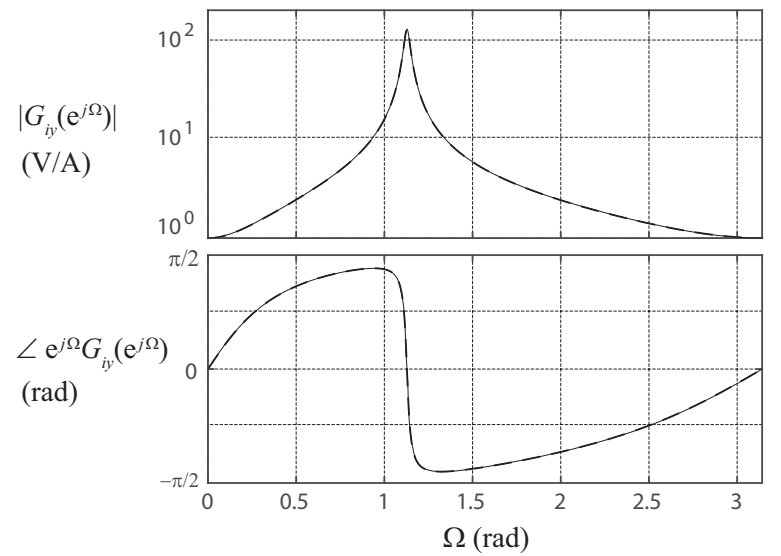

Figure 6: Transfer function $G_{i y}\left(e^{j \Omega}\right)$ (solid), and its finite-dimensional approximation (dashed)

For the example considered here, we assume $q(t)$ as in (4) is the only output available for feedback; i.e., $y_{k}=q_{k}$. This is the most extreme case, and performance can be expected to be better for cases in which additional outputs (such as heave position, wave height, etc.) are also available. We assume a time sample of $T=1$ s.

The resultant transfer function $G_{i y}\left(e^{j \omega}\right)$ is shown in Figure 6. (Actually, the figure shows $z G_{i y}\left(e^{j \Omega}\right)$, to verify that it is indeed positive real.) As can be seen, the heave resonance of the buoy has a natural period of approximately 6s. Recall that in this example, we presume this transfer function to be deterministic. A discrete-time approximate finite-dimensional model was obtained using a standard subspace algorithm, as was described in (McKelvey et al., 1996). The finite-dimensional model is shown as the dashed line in the plot. The finite-dimensional model has dimension $n_{1}=5$.

The open-circuit spectrum $\Sigma_{y 0}(\Omega)$ will vary, depending on the sea state parameters. For the purposes of demonstration, Figure 7 shows the spectrum for $T_{1}=9 \mathrm{~s}, \gamma=1$, and $H_{1 / 3}=1 \mathrm{~m}$. Throughout the course of the example, we assume that this spectrum can be adequately approximated by a spectral factor $U(z)$ of order $n=11$; i.e., we assume $x_{2}$ has dimension 6 . Note that although 


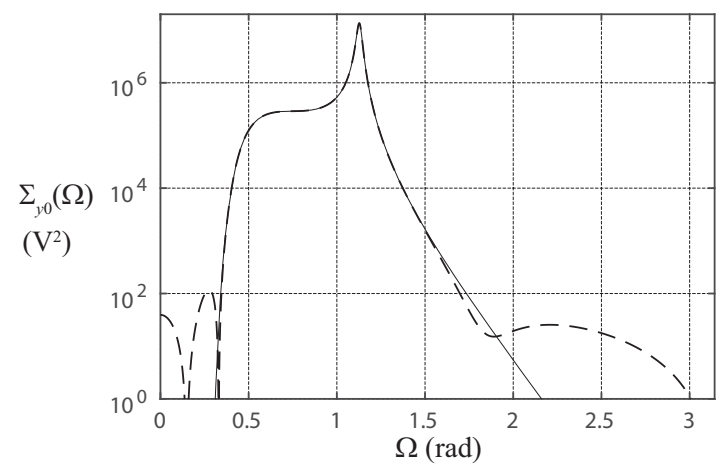

Figure 7: Example spectrum $\Sigma_{y 0}(\Omega)$ for sea state parameters $T_{1}=9 \mathrm{~s}, \gamma=1$, and $H_{1 / 3}=1 \mathrm{~m}$ (solid), and the finite-dimensional approximation (dashed)

we do solve for the wave excitation transfer function $f_{a}(j \omega)$ in order to simulate the buoy response, this transfer function is presumed to be unknown to the controller, a priori. To conduct the simulation, the spectral factor $U(z)$ is approximated from $\Sigma_{y 0}(\Omega)$ using a finite-dimensional spectral factorization algorithm. There are various computational techniques for performing spectral factorizations directly from nonparametric frequency-domain data. In this paper we make use of a subspace technique which was originally proposed in Van Overschee et al. (1997), although here we make use of a subsequent, more reliable technique proposed by Akcay (2011). We make no alterations to this algorithm, and consequently we will not go into explicit details on the technique here. However, Figure 7 shows both the true infinite-dimensional spectrum, as well as that of the finite-dimensional approximation with $n=11$.

To demonstrate the efficacy of the controller, we consider the following scenario. We assume that at time $k=0$, the buoy response is initiated, with the controller optimized for an assumed sea state characterized by $T_{1}=7 \mathrm{~s}, \gamma=3$, and $H_{1 / 3}=1.4 \mathrm{~m}$. However, in actuality the sea state has parameters $T_{1}=10 \mathrm{~s}$, $\gamma=1$, and $H_{1 / 3}=1.4 \mathrm{~m}$, and the controller must appropriately adapt $\tilde{\mathcal{H}}$ to correct for this initial detuning. We note that the incident wave power fluxes $p_{I}$ for the assumed and actual sea states are, respectively, 7.22 and $7.25 \mathrm{~kW} / \mathrm{m}$; i.e., they constitute sea states with nearly identical power flux. Meanwhile the 
theoretical causal power generation limits $\bar{p}_{0}$ for these two cases, as evaluated in (53), are $16.2 \mathrm{~kW}$ and $7.67 \mathrm{~kW}$ respectively. Furthermore, the theoretical power generation limits with the implementation of the sub-optimal observer, as in (71), are $15.6 \mathrm{~kW}$ and $7.43 \mathrm{~kW}$ respectively. It therefore stands to reason that the best performance achievable by the adaptive controller is $7.43 \mathrm{~kW}$, which would be attained by perfect identification of the true sea state.

For the purpose of demonstration, the controller is prohibited from adapting for a duration of one hour (i.e., $k=3600$ ), in order to illustrate the effect of the the de-tuning in sea state. The recursive estimation of $\hat{R}_{p}(k)$ for $p \in\{0 \ldots 99\}$ is also left dormant for $k<3600$, with the values frozen to be those for the assumed sea state. Following $k=3600$, the adaptation is initiated, including the recursive estimation of $R_{p}(k)$, as well as the adaptation of $\tilde{\mathcal{H}}$.

Figure 8 shows the resultant power output $p_{k}$, over the course of $10^{4}$ seconds (i.e., 2.78 hours) of system response. Also shown is the mean power generation $\bar{p}$ that the adaptive controller achieves in stationarity, which was calculated as the mean power over a stationary simulation of $10^{6} \mathrm{~s}$. We see that the adaptive controller is within $5 \%$ of achieving the theoretical upper power generation limit of $7.43 \mathrm{~kW}$. To achieve optimal performance, bi-directional power flow capability is required; i.e., the power take-off system must be capable of alternatingly extracting and injecting energy. Additionally, the magnitude of the power oscillations, about $\bar{p}$, are extreme. Indeed, this is an important feature of wave energy conversion - the power arrives in high pulsations, and must be smoothed significantly, prior to delivery to a utility. The intensity of these oscillations about $\bar{p}$ will generally be lower for lower values of $c_{e}$ (i.e., for a less efficient generator). However, in practice, an upper bound on the stationary variance on $p_{k}$ would likely need to be imposed as a competing objective in the control design.

To get a better idea for the performance of the adaptive controller, and the rate at which the adaptation occurs, it is useful to evaluate $\mathcal{E}\left\{p_{k}\right\}$ as a function of $k$. In the absence of analytical evolutionary equations for this expectation, it must be estimated via Monte Carlo analysis. Here, it has been evaluated by 


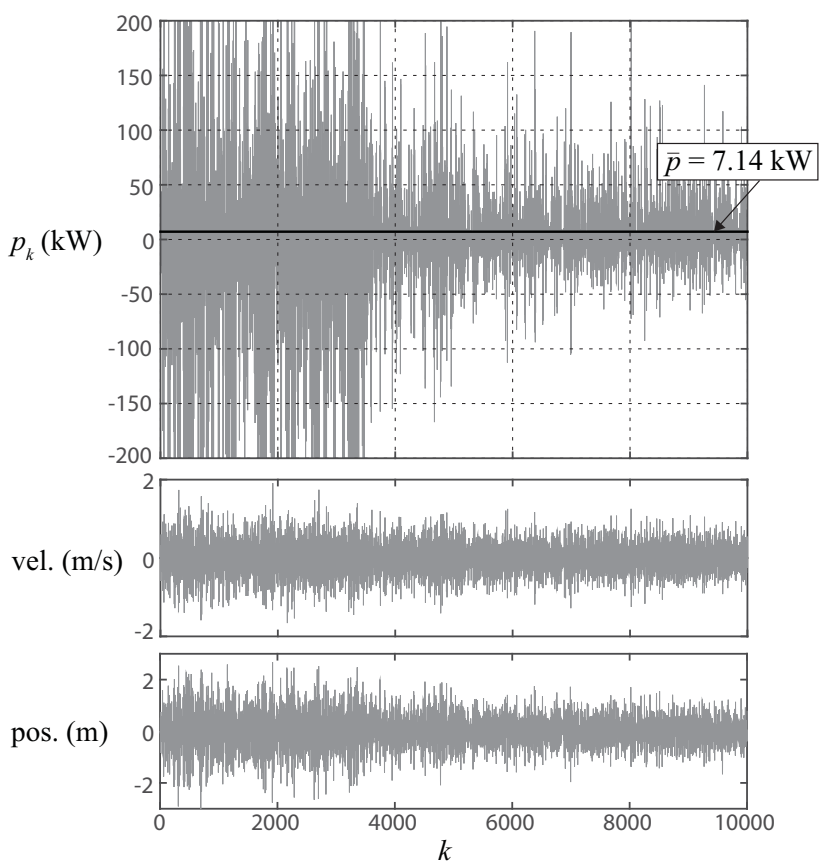

Figure 8: Example response (power generation, velocity, and position) of simulated buoy with adaptive control, with adaptation initiated at $k=3600$ 


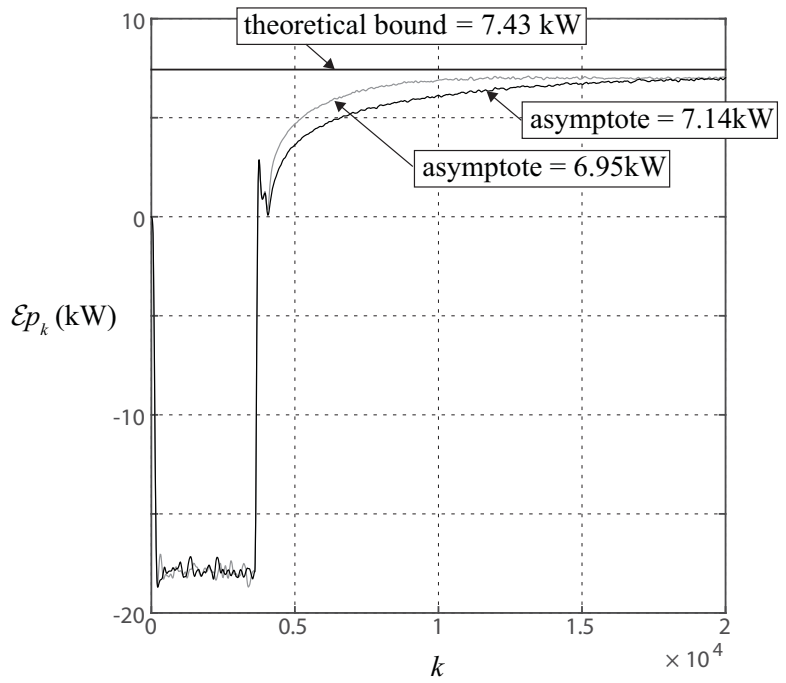

Figure 9: Monte Carlo estimate $\mathcal{E}\left\{p_{k}\right\}$ for $N_{\beta}=3600$ (black) and $N_{\beta}=1440$ (gray)

averaging over an ensemble of 5000 transient simulations for the same scenario as in Figure 8. The resultant ensemble average trajectory was then smoothed using a $3^{r d}$-order Butterworth lowpass filter with a cutoff frequency of $0.01 \mathrm{~Hz}$. The results are shown in Figure 9. From this plot, it is clear that the adaptive controller raises the mean power generation to a value close to its theoretical limit, and is also much clearer regarding the poor performance of the controller in its detuned state.

Also shown in Figure 9 is the analogous trajectory for $N_{\beta}=1440$ (i.e., 24 minutes). This reduction in $N_{\beta}$ allows the controller to adapt more quickly. However, the asymptotic performance achieved by the controller is lower than in the $N_{\beta}=3600$ case, because the accuracy of the identified disturbance model is less precise. As such, the plot illustrates the inherent trade-off in the problem, between the transient and asymptotic characteristics of the adaptive feedback law. 


\section{Conclusions}

It has only been very recently that the connection between stochastic energy harvesting and optimal causal stochastic control has been well understood. The fact that the optimal feedback law turns out to be the solution to an associated LQG problem motivates the use of the many existing techniques from this theory in energy harvesting problems. The aim of this paper has been to investigate the implications of indirect adaptive LQG control techniques, in this new context. The scope of the paper has been somewhat modest, in that we have focused only on the adaptive regulation problem, in which our knowledge of the plant is precise, while the knowledge of the disturbance is limited. Clearly, the next step is to examine the case in which the plant (i.e., transfer functions $Z_{i v}(s)$ and $\left.Z_{i y}(s)\right)$ are also unknown a priori. This case may pose considerably greater challenges.

We have focused the application of this paper on an ocean wave energy application, for a few reasons. Firstly, ocean energy applications provide strong incentive for the development of optimal control algorithms, as a means of maximizing the utililization of the available resource. Secondly, it constitutes an application for which the levels of generated power are large enough to offset the parasitic losses associated with the implementation of sophisticated control adaptation algorithms. This places WEC control in contrast to many smallerscale energy harvesting applications, such as energy scavengers for embedded sensing, which generate only a few milliwatts of power. Nonetheless, the control algorithms discussed in this paper may be of some use in some smaller-scale applications.

\section{Acknowledgments}

This work was supported by ONR award N000141010509 (Ron Joslin, program officer), and by NSF award 1235732. This funding is gratefully acknowledged. The views reported here are those of the authors, and do not necessarily reflect those of the sponsors. 


\section{References}

Akaike, H. (1975). Markovian representation of stochastic processes by canonical variables. SIAM Journal on Control and Optimization, 13, 162-173.

Akcay, H. (2011). Frequency domain subspace-based identification of discretetime power spectra from uniformly spaced measurements. Automatica, 47, 363-367.

Anderson, B. D. O., \& Vongpanitlerd, S. (1973). Network Analysis and Synthesis: A Modern Systems Theory Approach. Englewood Cliffs, NJ: PrenticeHall.

Bacelli, G., Gilloteaux, J., \& Ringwood, J. (2009). A predictive controller for a heaving buoy producing potable water. In Proceedings, 2009 European Control Conference (ECC) (pp. 3755-3760). IEEE.

Cretel, J. A. M., Lightbody, G., Thomas, G. P., \& Lewis, A. W. (2011). Maximisation of energy capture by a wave-energy point absorber using model predictive control. In Proceedings, $18^{\text {th }}$ IFAC World Congress. Milano.

Evans, D. V. (1981). Power from water waves. Annual Review of Fluid Mechan$i c s, 13,157-187$.

Falc̃ao, A. (2010). Wave energy utilization: A review of the technologies. Renewable and Sustainable Energy Reviews, 14, 899-918.

Falnes, J. (1980). Radiation impedance matrix and optimum power absorption for interacting oscillators in surface waves. Applied Ocean Research, 2, 75-80.

Falnes, J. (2002). Ocean Waves and Oscillating Systems, Linear Interaction including Wave Energy Extraction. Cambridge, UK: Cambridge University Press.

Faltinsen, O. M. (1990). Sea Loads on Ships and Offshore Structures. Cambridge University Press. 
Faurre, P. (1976). Stochastic realization algorithms. In R. K. Mehra, \& D. G. Lainiotis (Eds.), System Identification: Advances and Case Studies. New York: Academic Press.

Fusco, F., \& Ringwood, J. (2011). Suboptimal causal reactive control of wave energy converters using a second order system model. In Proceedings of the 21st (2011) International Offshore and Polar Engineering Conference (pp. 687-694). International Society of Offshore and Polar Engineers (ISOPE).

Fusco, F., \& Ringwood, J. V. (2010). Short-term wave forecasting for real-time control of wave energy converters. Sustainable Energy, IEEE Transactions on, 1, 99-106.

Goethals, I., Mevel, L., Benveniste, A., \& de Moor, B. (2004). Recursive outputonly subspace identification for in-flight flutter monitoring. In Proceedings, 22 ${ }^{\text {nd }}$ International Modal Analysis Conference.

Hals, J., Falnes, J., \& Moan, T. (2011a). A comparison of selected strategies for adaptive control of wave energy converters. Journal of Offshore Mechanics and Arctic Engineering, 133, 031101.

Hals, J., Falnes, J., \& Moan, T. (2011b). Constrained optimal control of a heaving buoy wave-energy converter. Journal of Offshore Mechanics and Arctic Engineering, 133, 011401.

Katayama, T. (2005). Subspace Methods for System Identification. London: Springer-Verlag.

Landau, I. D., Lozano, R., M'Saad, M., \& Karimi, A. (2011). Adaptive Control: Algorithms, Analysis and Applications. (2nd ed.). London: Springer-Verlag.

Leijon, M., Bernhoff, H., Agren, O., Isberg, J., Sundberg, J., Berg, M., Karlsson, K. E., \& Wolfbrandt, A. (2005). Multiphysics simulation of wave energy to electric energy conversion by permanent magnet linear generator. IEEE Transactions on Energy Conversion, 20, 219-224. 
Li, G., \& Belmont, M. R. (2014). Model predictive control of a sea wave energy converter: a convex approach. In at 19th IFAC World Congress, Cape Town, South Africa.

Mari, J., Stroica, P., \& McKelvey, T. (2000). Vector arma estimation: A reliable subspace approach. IEEE Transactions on Signal Processing, 48, 2092-2104.

McKelvey, T., Ackay, H., \& Ljung, L. (1996). Subspace-based identification of infinite-dimensional multivariable systems from frequency-response data. Automatica, 32, 885-902.

Mueller, M. A. (2002). Electrical generators for direct drive wave energy converters. IEE Proceedings, Part C, Generation, Transmission, 83 Distribution, 149 .

Nebel, P. (1992). Maximizing the efficiency of wave energy plant using complex conjugate control. Proceedings of the IME, Part I: Journal of Systems and Control Engineering, 206, 225-236.

Nie, R., \& Scruggs, J. T. (2014). Disturbance-adaptive stochastic optimal control of energy harvesters, with application to ocean wave energy conversion. In Proceedings, $19^{\text {th }}$ IFAC World Congress. Cape Town.

Polinder, H., Damen, E. C., \& Gardner, F. (2004). Linear PM generator system for wave energy conversion in the AWS. IEEE Transactions on Energy Conversion, 19, 583-589.

Richter, M., Magaña, M. E., Sawodny, O., \& Brekken, T. K. (2013). Nonlinear model predictive control of a point absorber wave energy converter. Sustainable Energy, IEEE Transactions on, 4, 118-126.

Ringwood, J. V., Baceli, G., \& Fusco, F. (2014). Energy-maximizing control of wave-energy converters: the development of control system technology to optimize their operation. IEEE Control Systems Magazine, 34, 30-55. 
Salter, S. H., Taylor, J. R. M., \& Caldwell, N. J. (2002). Power conversion mechanisms for wave energy. Proceedings of the IME, Part M: Journal of Engineering for the Maritime Environment, 216, 1-27.

Scruggs, J., Nie, R., Chertok, A., Clabby, D., Previsic, M., \& Karthikeyan, A. (2015). Optimal causal control of wave energy converters in stochastic waves - Accommodating nonlinear dynamic and loss models. In Proceedings of the 11th (2015) European Wave and Tidal Energy Conference. Nantes.

Scruggs, J. T. (2010). On the causal power generation limit for a vibratory energy harvester in broadband stochastic response. Journal of Intelligent Material Systems and Structures, 21, 1249-1262.

Scruggs, J. T., \& Behrens, S. (2011). Optimal Energy Harvesting From LowFrequency Bistate Force Loadings. ASME Journal of Vibration and Acoustics, 133.

Scruggs, J. T., Cassidy, I. L., \& Behrens, S. (2012). Multi-objective optimal control of vibratory energy harvesting systems. Journal of Intelligent Material Systems \& Structures, 23, 2077-2094.

Scruggs, J. T., Lattanzio, S. M., Taflanidis, A. A., \& Cassidy, I. L. (2013). Optimal causal control of an ocean wave energy converter in a random sea. Applied Ocean Research, 42, 1-15.

Van Overschee, P., De Moor, B., Dehandschutter, W., \& Swevers, J. (1997). A subspace algorithm for the identification of discrete time frequency domain power spectra. Automatica, 33, 2147-2157.

Xiao, C., \& Hill, D. (1999). Generalizations and new proof of the discrete-time positive real lemma and bounded real lemma. IEEE Transactions on Circuits and Systems I-Fundamental Theory and Applications, 46, 740-743. 